\title{
New Delay-Dependent Robust Exponential Stability Criteria of LPD Neutral Systems with Mixed Time-Varying Delays and Nonlinear Perturbations
}

\author{
Sirada Pinjai and Kanit Mukdasai \\ Department of Mathematics, Khon Kaen University, Khon Kaen 40002, Thailand \\ Correspondence should be addressed to Kanit Mukdasai; kanit@kku.ac.th
}

Received 15 July 2013; Revised 30 September 2013; Accepted 2 October 2013

Academic Editor: Jitao Sun

Copyright (C) 2013 S. Pinjai and K. Mukdasai. This is an open access article distributed under the Creative Commons Attribution License, which permits unrestricted use, distribution, and reproduction in any medium, provided the original work is properly cited.

\begin{abstract}
This paper is concerned with the problem of robust exponential stability for linear parameter-dependent (LPD) neutral systems with mixed time-varying delays and nonlinear perturbations. Based on a new parameter-dependent Lyapunov-Krasovskii functional, Leibniz-Newton formula, decomposition technique of coefficient matrix, free-weighting matrices, Cauchy's inequality, modified version of Jensen's inequality, model transformation, and linear matrix inequality technique, new delay-dependent robust exponential stability criteria are established in terms of linear matrix inequalities (LMIs). Numerical examples are given to show the effectiveness and less conservativeness of the proposed methods.
\end{abstract}

\section{Introduction}

Over the past decades, the problem of stability for neutral differential systems, which have delays in both their state and the derivatives of their states, has been widely investigated by many researchers, especially in the last decade. It is well known that nonlinearities, as time delays, may cause instability and poor performance of practical systems such as engineering, biology, and economics [1]. The problems of various stability and stabilization for dynamical systems with or without state delays and nonlinear perturbations have been intensively studied in the past years by many researchers of mathematics and control communities [1-35]. Stability criteria for dynamical systems with time delay are generally divided into two classes: delay-independent one and delaydependent one. Delay-independent stability criteria tend to be more conservative, especially for small size delay; such criteria do not give any information on the size of the delay. On the other hand, delay-dependent stability criteria are concerned with the size of the delay and usually provide a maximal delay size.
Recently, many researchers have studied the stability problem for neutral systems with time-varying delays and nonlinear perturbations have appeared [29, 31]. Furthermore, the convergence rates are essential for the practical system; then the exponential stability analysis of time delay systems has been favorably approved in the past decades; see, for example, $[3,9,10,14,18-21,25-28]$.

In addition, many researchers have paid attention to the problem of stability for linear systems with polytope uncertainties. The linear systems with polytopic-type uncertainties are called linear parameter-dependent (LPD) systems. That is, the uncertain state matrices are in the polytope consisting of all convex combination of known matrices. Most of sufficient (or necessary and sufficient) conditions have been obtained via Lyapunov-Krasovskii theory approaches in which parameter-dependent Lyapunov-Krasovskii functional has been employed. These conditions are always expressed in terms of linear matrix inequalities (LMIs). The results have been obtained for robust stability for LPD systems in which time-delay occurs in state variable; for example, $[17,18]$ presented sufficient conditions for robust 
stability of LPD discrete-time systems with delays. Moreover, robust stability of LPD continuous-time systems with delays was studied in $[6,19,22,30]$.

In consequence, it is important and interesting to study the problem of robust exponential stability for neutral systems with parametric uncertainties. This paper investigates the robust exponential stability analysis for LPD neutral systems with mixed time-varying delays and nonlinear perturbations. Based on combination of Leibniz-Newton formula, free-weighting matrices, Cauchy's inequality, modified version of Jensen's inequality, decomposition technique of coefficient matrix, the use of suitable parameter-dependent Lyapunov-Krasovskii functional, model transformation, and linear matrix inequality technique, new delay-dependent robust exponential stability criteria for these systems will be obtained in terms of LMIs. Finally, numerical examples will be given to show the effectiveness of the obtained results.

\section{Problem Formulation and Preliminaries}

We introduce some notations, a definition, and lemmas that will be used throughout the paper. $R^{+}$denotes the set of all real nonnegative numbers; $R^{n}$ denotes the $n$-dimensional space with the vector norm $\|\cdot\| ;\|x\|$ denotes the Euclidean vector norm of $x \in R^{n} ; R^{n \times r}$ denotes the set of $n \times r$ real matrices; $A^{T}$ denotes the transpose of the matrix $A$; $A$ is symmetric if $A=A^{T} ; I$ denotes the identity matrix; $\lambda(A)$ denotes the set of all eigenvalues of $A ; \lambda_{\max }(A)=$ $\max \{\operatorname{Re} \lambda: \lambda \in \lambda(A)\} ; \lambda_{\min }(A)=\min \{\operatorname{Re} \lambda: \lambda \in \lambda(A)\}$; $\lambda_{\text {max }}(A(\alpha))=\max \left\{\lambda_{\text {max }}\left(A_{i}\right): i=1,2, \ldots, N\right\} ; \lambda_{\text {min }}(A(\alpha))=$ $\min \left\{\lambda_{\min }\left(A_{i}\right): i=1,2, \ldots, N\right\} ; C\left([-b, 0], R^{n}\right)$ denotes the space of all continuous vector functions mapping $[-b, 0]$ into $R^{n}$, where $b=\max \{h, r\}, h, r \in R^{+} ; *$ represents the elements below the main diagonal of a symmetric matrix.

Consider the system described by the following state equations of the form:

$$
\begin{aligned}
\dot{x}(t)= & A(\alpha) x(t)+B(\alpha) x(t-h(t)) \\
& +C(\alpha) \dot{x}(t-r(t))+f(t, x(t)) \\
& +g(t, x(t-h(t)))+w(t, \dot{x}(t-r(t))), \quad t>0 ; \\
x\left(t+t_{0}\right) & =\phi(t), \quad \dot{x}\left(t+t_{0}\right)=\psi(t), \quad t \in[-b, 0],
\end{aligned}
$$

where $x(t) \in R^{n}$ is the state variable and $A(\alpha), B(\alpha), C(\alpha) \in$ $R^{n \times n}$ are uncertain matrices belonging to the polytope

$$
\begin{aligned}
A(\alpha)= & \sum_{i=1}^{N} \alpha_{i} A_{i}, \quad B(\alpha)=\sum_{i=1}^{N} \alpha_{i} B_{i}, \quad C(\alpha)=\sum_{i=1}^{N} \alpha_{i} C_{i} \\
& \sum_{i=1}^{N} \alpha_{i}=1, \quad \alpha_{i} \geq 0, A_{i}, B_{i}, C_{i} \in R^{n \times n}, i=1, \ldots, N
\end{aligned}
$$

$h(t)$ and $r(t)$ are discrete and neutral time-varying delays, respectively,

$$
\begin{array}{ll}
0 \leq h(t) \leq h, & \dot{h}(t) \leq h_{d}, \\
0 \leq r(t) \leq r, & \dot{r}(t) \leq r_{d},
\end{array}
$$

where $h, r, h_{d}$, and $r_{d}$ are given positive real constants. Consider the initial functions $\phi(t), \psi(t) \in C\left([-b, 0], R^{n}\right)$ with the norm $\|\phi\|=\sup _{t \in[-b, 0]}\|\phi(t)\|$ and $\|\psi\|=\sup _{t \in[-b, 0]}\|\psi(t)\|$. The uncertainties $f(t, x(t)), g(t, x(t-h(t)))$, and $w(t, \dot{x}(t-$ $r(t))$ ) are the nonlinear perturbations with respect to current state $x(t)$, discrete delayed state $x(t-h(t))$, and neutral delayed state $\dot{x}(t-r(t))$, respectively, and are bounded in magnitude:

$$
\begin{gathered}
f^{T}(t, x(t)) f(t, x(t)) \leq \eta^{2} x^{T}(t) x(t), \\
g^{T}(t, x(t-h(t))) g(t, x(t-h(t))) \\
\quad \leq \rho^{2} x^{T}(t-h(t)) x(t-h(t)), \\
w^{T}(t, \dot{x}(t-r(t))) w(t, \dot{x}(t-r(t))) \\
\quad \leq \gamma^{2} \dot{x}^{T}(t-r(t)) \dot{x}(t-r(t)),
\end{gathered}
$$

where $\eta, \rho$, and $\gamma$ are given positive real constants.

In order to improve the bound of the discrete timevarying delayed $h(t)$ in system (1), let us decompose the constant matrix $B(\alpha)$ as

$$
B(\alpha)=B_{1}(\alpha)+B_{2}(\alpha),
$$

where $B_{1}(\alpha)=\sum_{i=1}^{N} \alpha_{i} B_{i}^{1}, B_{2}(\alpha)=\sum_{i=1}^{N} \alpha_{i} B_{i}^{2}$, and $\sum_{i=1}^{N} \alpha_{i}=1$, $\alpha_{i} \geq 0$ with $B_{i}^{1}, B_{i}^{2} \in R^{n \times n}, i=1, \ldots, N$ being real constant matrices. By Leibniz-Newton formula, we have

$$
0=x(t)-x(t-h(t))-\int_{t-h(t)}^{t} \dot{x}(s) d s .
$$

By utilizing the following zero equation, we obtain

$$
0=G x(t)-G x(t-\beta h(t))-G \int_{t-\beta h(t)}^{t} \dot{x}(s) d s,
$$

where $\beta$ is a given positive real constant and $G \in R^{n \times n}$ will be chosen to guarantee the robust exponential stability of system (1). By (6), (7), and (8), system (1) can be represented by the form

$$
\begin{aligned}
\dot{x}(t)= & {\left[A(\alpha)+B_{1}(\alpha)+G\right] x(t)+B_{2}(\alpha) x(t-h(t)) } \\
& -G x(t-\beta h(t))+f(t, x(t)) \\
& +g(t, x(t-h(t)))+w(t, \dot{x}(t-r(t))) \\
& +C(\alpha) \dot{x}(t-r(t))-B_{1}(\alpha) \int_{t-h(t)}^{t} \dot{x}(s) d s \\
& -G \int_{t-\beta h(t)}^{t} \dot{x}(s) d s .
\end{aligned}
$$


Definition 1. The system (1) is robustly exponentially stable, if there exist positive real constants $k$ and $M$ such that, for each $\phi(t), \psi(t) \in C\left([-b, 0], R^{n}\right)$, the solution $x(t, \phi, \psi)$ of the system (1) satisfies

$$
\|x(t, \phi, \psi)\| \leq M \max \{\|\phi\|,\|\psi\|\} e^{-k t}, \quad \forall t \in R^{+} .
$$

Lemma 2 (Cauchy inequality). For any constant symmetric positive definite matrix $P \in R^{n \times n}$ and $a, b \in R^{n}$, one has

$$
\pm 2 a^{T} b \leq a^{T} P a+b^{T} P^{-1} b .
$$

Lemma 3 (see [15]). The following inequality holds for any $a \in$ $R^{n}, b \in R^{m}, N, Y \in R^{n \times m}, X \in R^{n \times n}$, and $Z \in R^{m \times m}$ :

$$
-2 a^{T} N b \leq\left[\begin{array}{l}
a \\
b
\end{array}\right]^{T}\left[\begin{array}{cc}
X & Y-N \\
* & Z
\end{array}\right]\left[\begin{array}{l}
a \\
b
\end{array}\right]
$$

where $\left[\begin{array}{ll}X & Y \\ * & Z\end{array}\right] \geq 0$.

Lemma 4. For any constant symmetric positive definite matrix $Q \in R^{n \times n}$ and $h(t)$ which is discrete time-varying delays with (3), vector function $\omega:[-h, 0] \rightarrow R^{n}$ such that the integrations concerned are well defined; then

$$
h \int_{-h}^{0} \omega^{T}(s) Q \omega(s) d s \geq \int_{-h(t)}^{0} \omega^{T}(s) d s Q \int_{-h(t)}^{0} \omega(s) d s .
$$

Proof. From Lemma 2, it is easy to see that

$$
\begin{aligned}
h \int_{-h}^{0} \omega^{T}(s) Q \omega(s) d s= & h \int_{-h(t)}^{0} \omega^{T}(s) Q \omega(s) d s \\
& +h \int_{-h}^{-h(t)} \omega^{T}(s) Q \omega(s) d s \\
\geq & h(t) \int_{-h(t)}^{0} \omega^{T}(s) Q \omega(s) d s \\
= & \frac{1}{2} \iint_{-h(t)}^{0}\left[\omega^{T}(s) Q \omega(s)\right. \\
\geq & \frac{1}{2} \iint_{-h(t)}^{0} 2 \omega^{T}(s) Q^{1 / 2^{T}} \\
= & \int_{-h(t)}^{0} \omega^{T}(s) d s Q \int_{-h(t)}^{0} \omega(s) d s .
\end{aligned}
$$

Lemma 5. Let $x(t) \in R^{n}$ be a vector-valued function with first order continuous derivative entries. Then, the following integral inequality holds for any matrices $M_{i} \in R^{n \times n}, i=1,2, \ldots, 5$, and $h(t)$ is discrete time-varying delays with (3) and symmetric positive definite matrix $X \in R^{n \times n}$ :

$$
\begin{aligned}
-\int_{t-h}^{t} \dot{x}^{T}(s) X \dot{x}(s) d s \leq & {\left[\begin{array}{c}
x(t) \\
x(t-h(t))
\end{array}\right]^{T} } \\
& \times\left[\begin{array}{cc}
M_{1}+M_{1}^{T} & -M_{1}^{T}+M_{2} \\
* & -M_{2}-M_{2}^{T}
\end{array}\right] \\
& \times\left[\begin{array}{c}
x(t) \\
x(t-h(t))
\end{array}\right] \\
& +h\left[\begin{array}{c}
x(t) \\
x(t-h(t))
\end{array}\right]^{T}\left[\begin{array}{cc}
M_{3} & M_{4} \\
* & M_{5}
\end{array}\right] \\
& \times\left[\begin{array}{c}
x(t) \\
x(t-h(t))
\end{array}\right],
\end{aligned}
$$

where

$$
\left[\begin{array}{ccc}
X & M_{1} & M_{2} \\
* & M_{3} & M_{4} \\
* & * & M_{5}
\end{array}\right] \geq 0
$$

Proof. From the Leibniz-Newton formula, one has

$$
0=x(t)-x(t-h(t))-\int_{t-h(t)}^{t} \dot{x}(t) d s .
$$

Therefore, for any $H_{1}, H_{2} \in R^{n \times n}$, the following equation is true:

$$
\begin{aligned}
& 0=2\left[x^{T}(t)-x^{T}(t-h(t))-\int_{t-h(t)}^{t} \dot{x}^{T}(t) d s\right] \\
& \times\left[H_{1} x(t)+H_{2} x(t-h(t))\right] \\
& =2 x^{T}(t) H_{1} x(t)+2 x^{T}(t) H_{2} x(t-h(t)) \\
& -2 x^{T}(t-h(t)) H_{1} x(t) \\
& -2 x^{T}(t-h(t)) H_{2}^{T} x(t-h(t)) \\
& -2 \int_{t-h(t)}^{t} \dot{x}^{T}(s) d s H_{1} x(t) \\
& -2 \int_{t-h(t)}^{t} \dot{x}^{T}(s) d s H_{2} x(t-h(t)) \\
& =\left[\begin{array}{c}
x(t) \\
x(t-h(t))
\end{array}\right]^{T}\left[\begin{array}{cc}
H_{1}+H_{1}^{T} & -H_{1}^{T}+H_{2} \\
* & -H_{2}-H_{2}^{T}
\end{array}\right] \\
& \times\left[\begin{array}{c}
x(t) \\
x(t-h(t))
\end{array}\right] \\
& -2 \int_{t-h(t)}^{t} \dot{x}^{T}(s)\left[\begin{array}{ll}
H_{1} & H_{2}
\end{array}\right]\left[\begin{array}{c}
x(t) \\
x(t-h(t))
\end{array}\right] d s .
\end{aligned}
$$


Using Lemma 3 with $a=\dot{x}(s), b=\left[\begin{array}{c}x(t) \\ x(t-h(t))\end{array}\right], N=\left[H_{1}\right.$ $\left.H_{2}\right], Y=\left[\begin{array}{ll}M_{1} & M_{2}\end{array}\right]$, and $Z=\left[\begin{array}{cc}M_{3} & M_{4} \\ * & M_{5}\end{array}\right]$, we obtain

$$
\begin{aligned}
& -2 \int_{t-h(t)}^{t} \dot{x}^{T}(s)\left[\begin{array}{ll}
H_{1} & H_{2}
\end{array}\right]\left[\begin{array}{c}
x(t) \\
x(t-h(t))
\end{array}\right] d s \\
& \leq \int_{t-h(t)}^{t}\left[\begin{array}{c}
\dot{x}(s) \\
x(t) \\
x(t-h(t))
\end{array}\right]^{T}\left[\begin{array}{ccc}
X & M_{1}-H_{1} & M_{2}-H_{2} \\
* & M_{3} & M_{4} \\
* & * & M_{5}
\end{array}\right] \\
& \times\left[\begin{array}{c}
\dot{x}(s) \\
x(t) \\
x(t-h(t))
\end{array}\right] d s \\
& =\int_{t-h(t)}^{t} \dot{x}^{T}(s) X \dot{x}(s) d s \\
& +\left[\begin{array}{c}
x(t) \\
x(t-h(t))
\end{array}\right]^{T} \\
& \times\left[\begin{array}{cc}
M_{1}+M_{1}^{T}-H_{1}-H_{1}^{T} & -M_{1}^{T}+M_{2}+H_{1}^{T}-H_{2} \\
* & -M_{2}-M_{2}^{T}+H_{2}+H_{2}^{T}
\end{array}\right] \\
& \times\left[\begin{array}{c}
x(t) \\
x(t-h(t))
\end{array}\right]+h(t)\left[\begin{array}{c}
x(t) \\
x(t-h(t))
\end{array}\right]^{T}\left[\begin{array}{cc}
M_{3} & M_{4} \\
* & M_{5}
\end{array}\right] \\
& \times\left[\begin{array}{c}
x(t) \\
x(t-h(t))
\end{array}\right] \\
& \leq \int_{t-h(t)}^{t} \dot{x}^{T}(s) X \dot{x}(s) d s+\left[\begin{array}{c}
x(t) \\
x(t-h(t))
\end{array}\right]^{T} \\
& \times\left(\left[\begin{array}{cc}
M_{1}+M_{1}^{T} & -M_{1}^{T}+M_{2} \\
* & -M_{2}-M_{2}^{T}
\end{array}\right]\right. \\
& \left.-\left[\begin{array}{cc}
H_{1}+H_{1}^{T} & -H_{1}^{T}+H_{2} \\
* & -H_{2}-H_{2}^{T}
\end{array}\right]\right)\left[\begin{array}{c}
x(t) \\
x(t-h(t))
\end{array}\right] \\
& +h\left[\begin{array}{c}
x(t) \\
x(t-h(t))
\end{array}\right]^{T}\left[\begin{array}{cc}
M_{3} & M_{4} \\
* & M_{5}
\end{array}\right]\left[\begin{array}{c}
x(t) \\
x(t-h(t))
\end{array}\right] .
\end{aligned}
$$

Substituting (19) into (18), we obtain

$$
\begin{aligned}
-\int_{t-h(t)}^{t} \dot{x}^{T}(s) X \dot{x}(s) d s \leq & {\left[\begin{array}{c}
x(t) \\
x(t-h(t))
\end{array}\right]^{T} } \\
& \times\left[\begin{array}{cc}
H_{1}+H_{1}^{T} & -H_{1}^{T}+H_{2} \\
* & -H_{2}-H_{2}^{T}
\end{array}\right] \\
& \times\left[\begin{array}{c}
x(t) \\
x(t-h(t))
\end{array}\right]
\end{aligned}
$$

$$
\begin{aligned}
& +\left[\begin{array}{c}
x(t) \\
x(t-h(t))
\end{array}\right]^{T} \\
& \times\left(\left[\begin{array}{cc}
M_{1}+M_{1}^{T} & -M_{1}^{T}+M_{2} \\
* & -M_{2}-M_{2}^{T}
\end{array}\right]\right. \\
& \text { - } \left.\left[\begin{array}{cc}
H_{1}+H_{1}^{T} & -H_{1}^{T}+H_{2} \\
* & -H_{2}-H_{2}^{T}
\end{array}\right]\right) \\
& \times\left[\begin{array}{c}
x(t) \\
x(t-h(t))
\end{array}\right] \\
& +h\left[\begin{array}{c}
x(t) \\
x(t-h(t))
\end{array}\right]^{T}\left[\begin{array}{cc}
M_{3} & M_{4} \\
* & M_{5}
\end{array}\right] \\
& \times\left[\begin{array}{c}
x(t) \\
x(t-h(t))
\end{array}\right] \\
& =\left[\begin{array}{c}
x(t) \\
x(t-h(t))
\end{array}\right]^{T} \\
& \times\left[\begin{array}{cc}
M_{1}+M_{1}^{T} & -M_{1}^{T}+M_{2} \\
* & -M_{2}-M_{2}^{T}
\end{array}\right] \\
& \times\left[\begin{array}{c}
x(t) \\
x(t-h(t))
\end{array}\right] \\
& +h\left[\begin{array}{c}
x(t) \\
x(t-h(t))
\end{array}\right]^{T}\left[\begin{array}{cc}
M_{3} & M_{4} \\
* & M_{5}
\end{array}\right] \\
& \times\left[\begin{array}{c}
x(t) \\
x(t-h(t))
\end{array}\right] \text {. }
\end{aligned}
$$

From (3), it is clear that

$$
-\int_{t-h}^{t} \dot{x}^{T}(s) X \dot{x}(s) d s \leq-\int_{t-h(t)}^{t} \dot{x}^{T}(s) X \dot{x}(s) d s .
$$

From (20) and (21), the integral inequality becomes

$$
\begin{aligned}
-\int_{t-h}^{t} \dot{x}^{T}(s) X \dot{x}(s) d s \leq & {\left[\begin{array}{c}
x(t) \\
x(t-h(t))
\end{array}\right]^{T} } \\
& \times\left[\begin{array}{cc}
M_{1}+M_{1}^{T} & -M_{1}^{T}+M_{2} \\
* & -M_{2}-M_{2}^{T}
\end{array}\right] \\
& \times\left[\begin{array}{c}
x(t) \\
x(t-h(t))
\end{array}\right] \\
& +h\left[\begin{array}{c}
x(t) \\
x(t-h(t))
\end{array}\right]^{T}\left[\begin{array}{cc}
M_{3} & M_{4} \\
* & M_{5}
\end{array}\right] \\
& \times\left[\begin{array}{c}
x(t) \\
x(t-h(t))
\end{array}\right] .
\end{aligned}
$$

The proof of the theorem is complete. 
Remark 6. In Lemma 4 and Lemma 5, we have modified the method from $[8,33]$, respectively.

\section{Main results}

3.1. Robust Exponential Stability Criteria. In this section, robust exponential stability criteria dependent on mixed time-varying delays of LPD neutral delayed system (1) with nonlinear perturbations via linear matrix inequality (LMI) approach will be presented. We introduce the following notations for later use:

$$
\begin{aligned}
& P_{j}(\alpha)=\sum_{i=1}^{N} \alpha_{i} P_{i}^{j}, \quad Z_{1}(\alpha)=\sum_{i=1}^{N} \alpha_{i} Z_{i}^{1}, \\
& R_{p}(\alpha)=\sum_{i=1}^{N} \alpha_{i} R_{i}^{p}, \quad N_{j}(\alpha)=\sum_{i=1}^{N} \alpha_{i} N_{i}^{j}, \\
& O_{j}(\alpha)=\sum_{i=1}^{N} \alpha_{i} O_{i}^{j}, \quad W_{j}(\alpha)=\sum_{i=1}^{N} \alpha_{i} W_{i}^{j}, \\
& M_{j}(\alpha)=\sum_{i=1}^{N} \alpha_{i} M_{i}^{j}, \quad \sum_{i=1}^{N} \alpha_{i}=1, \quad \alpha_{i} \geq 0, \\
& P_{i}^{j}, Z_{i}^{1}, R_{i}^{p}, W_{i}^{j}, N_{i}^{j}, O_{i}^{j}, M_{i}^{j} \in R^{n \times n}, \\
& j=1,2, \ldots, 10, p=1,2, \ldots, 6, i=1,2, \ldots, N \text {; } \\
& \sum_{i, j}
\end{aligned}
$$

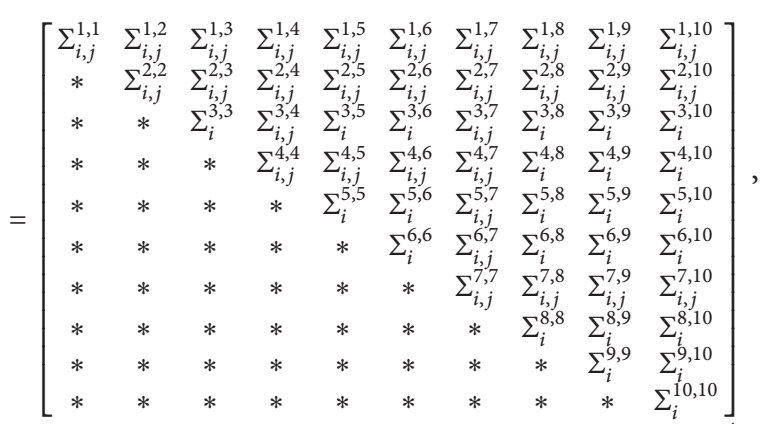

where

$$
\begin{aligned}
\Sigma_{i, j}^{1,1}= & A_{i}^{T} P_{j}^{1}+B_{i}^{1^{T}} P_{j}^{1}+P_{i}^{1} A_{j}+P_{i}^{1} B_{j}^{1}+Z_{i}^{1}+Z_{i}^{1^{T}} \\
& +P_{i}^{3}+P_{i}^{4}+h M_{i}^{1^{T}}+h M_{i}^{1}-e^{-2 k h} P_{i}^{9} \\
& -e^{-2 k \beta h} P_{i}^{10}+h^{2} M_{i}^{3}+\beta h M_{i}^{6}+\beta h M_{i}^{6^{T}}+\beta^{2} h^{2} M_{i}^{8} \\
& +N_{i}^{1^{T}}+N_{i}^{1}+O_{i}^{1^{T}}+O_{i}^{1}+A_{i}^{T} W_{j}^{1}+W_{i}^{1^{T}} A_{j} \\
& +B_{i}^{1^{T}} W_{j}^{1}+W_{i}^{1^{T}} B_{j}^{1}+\epsilon_{1} \eta^{2} I+2 k P_{i}^{1},
\end{aligned}
$$

$$
\begin{aligned}
& \Sigma_{i, j}^{1,2}=P_{i}^{1} B_{j}^{2}-h M_{i}^{1^{T}}+h M_{i}^{2}+h^{2} M_{i}^{4}+e^{-2 k h} P_{i}^{9} \\
& +h R_{i}^{2}-N_{i}^{1^{T}}+N_{i}^{2}+O_{i}^{2}+W_{i}^{1^{T}} B_{j}^{2} \\
& +A_{i}^{T} W_{j}^{2}+B_{i}^{1^{T}} W_{j}^{2}, \\
& \Sigma_{i, j}^{1,3}=-Z_{i}^{1}-\beta h M_{i}^{6^{T}}+\beta h M_{i}^{7}+\beta h^{2} M_{i}^{9}+\beta h R_{i}^{5} \\
& +e^{-2 k \beta h} P_{i}^{10}+N_{i}^{3}-O_{i}^{1^{T}}+O_{i}^{3} \\
& +A_{i}^{T} W_{j}^{3}+B_{i}^{1^{T}} W_{j}^{3}, \\
& \Sigma_{i, j}^{1,4}=-P_{i}^{1} B_{j}^{1}-N_{i}^{1^{T}}+N_{i}^{4}+O_{i}^{4} \\
& -W_{i}^{1^{T}} B_{j}^{1}+A_{i}^{T} W_{j}^{4}+B_{i}^{1^{T}} W_{j}^{4}, \\
& \Sigma_{i, j}^{1,5}=-Z_{i}^{1}+N_{i}^{5}-O_{i}^{1^{T}}+O_{i}^{5}+A_{i}^{T} W_{j}^{5}+B_{i}^{1^{T}} W_{j}^{5}, \\
& \Sigma_{i, j}^{1,6}=N_{i}^{6}+O_{i}^{6}-W_{i}^{1^{T}}+A_{i}^{T} W_{j}^{6}+B_{i}^{1^{T}} W_{j}^{6}, \\
& \Sigma_{i, j}^{1,7}=P_{i}^{1} C_{j}+N_{i}^{7}+O_{i}^{7}+W_{i}^{1^{T}} C_{j}+A_{i}^{T} W_{j}^{7}+B_{i}^{1^{T}} W_{j}^{7}, \\
& \Sigma_{i, j}^{1,8}=P_{i}^{1}+N_{i}^{8}+O_{i}^{8}+W_{i}^{1^{T}}+A_{i}^{T} W_{j}^{8}+B_{i}^{1^{T}} W_{j}^{8}, \\
& \Sigma_{i, j}^{1,9}=P_{i}^{1}+N_{i}^{9}+O_{i}^{9}+W_{i}^{1^{T}}+A_{i}^{T} W_{j}^{9}+B_{i}^{1^{T}} W_{j}^{9}, \\
& \Sigma_{i, j}^{1,10}=P_{i}^{1}+N_{i}^{10}+O_{i}^{10}+W_{i}^{1^{T}}+A_{i}^{T} W_{j}^{10}+B_{i}^{1^{T}} W_{j}^{10}, \\
& \Sigma_{i, j}^{2,2}=-\left(1-h_{d}\right) e^{-2 k h} P_{i}^{3}-h M_{i}^{2^{T}}-h M_{i}^{2}+h^{2} M_{i}^{5} \\
& +h^{2} R_{i}^{1}-2 h R_{i}^{2}-e^{-2 k h} P_{i}^{9}+\epsilon_{2} \rho^{2} I-N_{i}^{2^{T}} \\
& -N_{i}^{2}+W_{i}^{2^{T}} B_{j}^{2}+B_{i}^{2^{T}} W_{j}^{2}, \\
& \Sigma_{i, j}^{2,3}=-N_{i}^{3}-O_{i}^{2^{T}}+B_{i}^{2^{T}} W_{j}^{3}, \\
& \Sigma_{i, j}^{2,4}=-N_{i}^{2^{T}}-N_{i}^{4}-W_{i}^{2^{T}} B_{j}^{1}+B_{i}^{2^{T}} W_{j}^{4}, \\
& \Sigma_{i, j}^{2,5}=-N_{i}^{5}-O_{i}^{2^{T}}+B_{i}^{2^{T}} W_{j}^{5}, \\
& \Sigma_{i, j}^{2,6}=-N_{i}^{6}-W_{i}^{2^{T}}+B_{i}^{2^{T}} W_{j}^{6}, \\
& \Sigma_{i, j}^{2,7}=-N_{i}^{7}+W_{i}^{2^{T}} C_{j}+B_{i}^{2^{T}} W_{j}^{7}, \\
& \Sigma_{i, j}^{2,8}=-N_{i}^{8}+W_{i}^{2^{T}}+B_{i}^{2^{T}} W_{j}^{8}, \\
& \Sigma_{i, j}^{2,9}=-N_{i}^{9^{T}}-W_{i}^{2^{T}}+B_{i}^{2^{T}} W_{j}^{9}, \\
& \Sigma_{i, j}^{2,10}=-N_{i}^{10}+W_{i}^{2^{T}}+B_{i}^{2^{T}} W_{j}^{10}, \\
& \Sigma_{i}^{3,3}=-\left(1-\beta h_{d}\right) e^{-2 \beta k h} P_{i}^{4}-e^{-2 \beta k h} P_{i}^{10}+\beta^{2} h^{2} R_{i}^{4} \\
& -2 \beta h R_{i}^{5}-\beta h M_{i}^{7^{T}}-\beta h M_{i}^{7}+\beta^{2} h^{2} M_{i}^{10} \\
& -O_{i}^{3^{T}}-O_{i}^{3}
\end{aligned}
$$




$$
\begin{aligned}
& \Sigma_{i, j}^{3,4}=-N_{i}^{3^{T}}-O_{i}^{4}-W_{i}^{3^{T}} B_{j}^{1} \\
& \Sigma_{i}^{3,5}=-O_{i}^{3^{T}}-O_{i}^{5}, \quad \Sigma_{i}^{3,6}=-O_{i}^{9}+W_{i}^{3^{T}}, \\
& \Sigma_{i}^{3,7}=-O_{i}^{7}+W_{i}^{3^{T}} C_{j}, \quad \Sigma_{i}^{3,8}=-O_{i}^{8}+W_{i}^{3^{T}}, \\
& \Sigma_{i}^{3,9}=-O_{i}^{9}+W_{i}^{3^{T}}, \quad \Sigma_{i}^{3,10}=-O_{i}^{10}+W_{i}^{3^{T}}, \\
& \Sigma_{i, j}^{4,4}=-e^{2 k h} P_{i}^{7}-N_{i}^{4^{T}}-N_{i}^{4}+W_{i}^{4^{T}} B_{j}^{1}-B_{i}^{1^{T}} W_{j}^{4}, \\
& \Sigma_{i, j}^{4,5}=-N_{i}^{5}-O_{i}^{4^{T}}-B_{i}^{1^{T}} W_{j}^{5}, \\
& \Sigma_{i, j}^{4,6}=-N_{i}^{6}-W_{i}^{4^{T}}-B_{i}^{1^{T}} W_{j}^{6}, \\
& \Sigma_{i, j}^{4,7}=-N_{i}^{7}+W_{i}^{4^{T}} C_{j}-B_{i}^{1^{T}} W_{j}^{7}, \quad \Sigma_{i}^{4,8}=-O_{i}^{8}+W_{i}^{3^{T}}, \\
& \Sigma_{i}^{4,9}=-O_{i}^{9}+W_{i}^{3^{T}}, \quad \Sigma_{i}^{4,10}=-O_{i}^{10}+W_{i}^{3^{T}}, \\
& \Sigma_{i}^{5,5}=-e^{2 \beta k h} P_{i}^{8}-O_{i}^{5^{T}}-O_{i}^{5}, \quad \Sigma_{i}^{5,6}=-O_{i}^{6}-W_{i}^{5^{T}}, \\
& \Sigma_{i, j}^{5,7}=-O_{i}^{7}+W_{i}^{5^{T}} C_{j}, \quad \Sigma_{i}^{5,8}=-O_{i}^{8}+W_{i}^{5^{T}}, \\
& \Sigma_{i}^{5,9}=-O_{i}^{9}+W_{i}^{5^{T}}, \quad \Sigma_{i}^{5,10}=-O_{i}^{10}+W_{i}^{5^{T}}, \\
& \Sigma_{i}^{6,6}=P_{i}^{2}+h^{2} P_{i}^{5}+\beta^{2} h^{2} P_{i}^{6}+h^{2} P_{i}^{7}+\beta^{2} h^{2} P_{i}^{8} \\
& +h^{2} P_{i}^{9}+\beta^{2} h^{2} P_{i}^{10}-W_{i}^{6^{T}}-W_{i}^{6}, \\
& \Sigma_{i, j}^{6,7}=W_{i}^{6^{T}} C_{j}-W_{i}^{7}, \quad \Sigma_{i}^{6,8}=W_{i}^{6^{T}}-W_{i}^{8}, \\
& \Sigma_{i}^{6,9}=W_{i}^{6^{T}}-W_{i}^{9}, \quad \Sigma_{i}^{6,10}=W_{i}^{6^{T}}-W_{i}^{10}, \\
& \Sigma_{i, j}^{7,7}=-\left(1-r_{d}\right) e^{-2 k r} P_{i}^{2}+\epsilon_{3} \gamma^{2} I+W_{i}^{7^{T}} C_{j}+C_{i}^{T} W_{j}^{7}, \\
& \Sigma_{i, j}^{7,8}=W_{i}^{7^{T}}-C_{i}^{T} W_{j}^{8}, \quad \Sigma_{i, j}^{7,9}=W_{i}^{7^{T}}-C_{i}^{T} W_{j}^{9}, \\
& \Sigma_{i, j}^{7,10}=W_{i}^{7^{T}}-C_{i}^{T} W_{j}^{10}, \quad \Sigma_{i}^{8,8}=W_{i}^{8^{T}}+W_{i}^{8}-\epsilon_{1} I, \\
& \Sigma_{i}^{8,9}=W_{i}^{8^{T}}+W_{i}^{9}, \quad \Sigma_{i}^{8,10}=W_{i}^{8^{T}}+W_{i}^{10}, \\
& \Sigma_{i}^{9,9}=W_{i}^{9^{T}}+W_{i}^{9}-\epsilon_{2} I, \quad \Sigma_{i}^{9,10}=W_{i}^{9^{T}}+W_{i}^{10}, \\
& \Sigma_{i}^{10,10}=W_{i}^{10^{T}}+W_{i}^{10}-\epsilon_{3} I, \\
& \widehat{\Sigma_{i}^{7,7}}=-(1-r d) e^{2 k r} P_{i}^{2}+W_{i}^{7^{T}} C_{j}+C_{i}^{T} W_{j}^{7}, \\
& Z_{i}^{1}=P_{i}^{1} G
\end{aligned}
$$

Theorem 7. For $\left\|C_{i}\right\|+\gamma<1, i=1,2, \ldots, N$ and given positive real constants $h, h_{d}, r, r_{d}, \eta, \rho, \gamma$, and $\beta$, system (1) is robustly exponentially stable with a decay rate $k$, if there exist symmetric positive definite matrices $P_{i}^{j}$, any appropriate dimensional matrices $R_{i}^{p}, N_{i}^{j}, O_{i}^{j}, W_{i}^{j}, M_{i}^{j}, G, p=1,2, \ldots 6$, $j=1,2, \ldots, 10, i=1,2, \ldots, N$, and positive real constants $\epsilon_{1}, \epsilon_{2}$, and $\epsilon_{3}$ such that the following symmetric linear matrix inequalities hold:

$$
\begin{aligned}
& {\left[\begin{array}{cc}
R_{i}^{1} & R_{i}^{2} \\
* & R_{i}^{3}
\end{array}\right]>0, \quad i=1,2, \ldots, N} \\
& {\left[\begin{array}{cc}
R_{i}^{4} & R_{i}^{5} \\
* & R_{i}^{6}
\end{array}\right]>0, \quad i=1,2, \ldots, N \text {, }} \\
& {\left[\begin{array}{ccc}
e^{-2 k h} P_{i}^{3}-R_{i}^{3} & M_{i}^{1} & M_{i}^{2} \\
* & M_{i}^{3} & M_{i}^{4} \\
* & * & M_{i}^{5}
\end{array}\right] \geq 0, \quad i=1,2, \ldots, N,} \\
& {\left[\begin{array}{ccc}
e^{-2 \beta k h} P_{i}^{6}-R_{i}^{6} & M_{i}^{6} & M_{i}^{7} \\
* & M_{i}^{8} & M_{i}^{9} \\
* & * & M_{i}^{10}
\end{array}\right] \geq 0, \quad i=1,2, \ldots, N} \\
& \sum_{i, i}<-I, \quad i=1,2, \ldots, N \\
& \sum_{i, j}+\sum_{j, i}<\frac{2}{(N-1)} I, \quad i=1,2, \ldots, N-1, \\
& j=i+1, i+2, \ldots, N \text {. }
\end{aligned}
$$

Moreover, the solution $x(t, \phi, \psi)$ satisfies the inequality

$$
\|x(t, \phi, \psi)\| \leq \sqrt{\frac{L}{\lambda_{\min }\left(P_{1}(\alpha)\right)}} \max [\|\phi\|,\|\psi\|] e^{-k t},
$$

$\forall t \in R^{+}$

where $L=\lambda_{\max }\left(P_{1}(\alpha)\right)+r \lambda_{\max }\left(P_{2}(\alpha)\right)+h \lambda_{\max }\left(P_{3}(\alpha)\right)+$ $\beta h \lambda_{\max }\left(P_{4}(\alpha)\right)+h^{3} \lambda_{\max }\left(P_{5}(\alpha)+P_{7}(\alpha)+P_{9}(\alpha)\right)+$ $(\beta h)^{3} \lambda_{\text {max }}\left(P_{6}(\alpha)+P_{8}(\alpha)+P_{10}(\alpha)\right)+h^{3} \lambda_{\max }\left(\left[\begin{array}{cc}R_{1}(\alpha) & R_{2}(\alpha) \\ * & R_{3}(\alpha)\end{array}\right]\right)+$ $(\beta h)^{3} \lambda_{\max }\left(\left[\begin{array}{cc}R_{4}(\alpha) & R_{5}(\alpha) \\ * & R_{6}(\alpha)\end{array}\right]\right)$.

Proof. Choose a parameter-dependent Lyapunov-Krasovskii functional candidate for system (9) as

$$
V(t)=\sum_{i=1}^{7} V_{i}(t)
$$

where

$$
\begin{gathered}
V_{1}(t)=x^{T}(t) P_{1}(\alpha) x(t), \\
V_{2}(t)=\int_{t-r(t)}^{t} e^{2 k(s-t)} \dot{x}^{T}(s) P_{2}(\alpha) \dot{x}(s) d s, \\
V_{3}(t)=\int_{t-h(t)}^{t} e^{2 k(s-t)} x^{T}(s) P_{3}(\alpha) x(s) d s \\
+\int_{t-\beta h(t)}^{t} e^{2 k(s-t)} x^{T}(s) P_{4}(\alpha) x(s) d s,
\end{gathered}
$$




$$
\begin{aligned}
& V_{4}(t)=h \int_{-h}^{0} \int_{t+\theta}^{t} e^{2 k(s-t)} \dot{x}^{T}(s) P_{5}(\alpha) \dot{x}(s) d s d \theta \\
& +\beta h \int_{-\beta h}^{0} \int_{t+\theta}^{t} e^{2 k(s-t)} \dot{x}^{T}(s) P_{6}(\alpha) \dot{x}(s) d s d \theta, \\
& V_{5}(t)=h \int_{-h}^{0} \int_{t+\theta}^{t} e^{2 k(s-t)} \dot{x}^{T}(s) P_{7}(\alpha) \dot{x}(s) d s d \theta \\
& +\beta h \int_{-\beta h}^{0} \int_{t+\theta}^{t} e^{2 k(s-t)} \dot{x}^{T}(s) P_{8}(\alpha) \dot{x}(s) d s d \theta, \\
& V_{6}(t)=h \int_{-h}^{0} \int_{t+\theta}^{t} e^{2 k(s-t)} \dot{x}^{T}(s) P_{9}(\alpha) \dot{x}(s) d s d \theta \\
& +\beta h \int_{-\beta h}^{0} \int_{t+\theta}^{t} e^{2 k(s-t)} \dot{x}^{T}(s) P_{10}(\alpha) \dot{x}(s) d s d \theta, \\
& V_{7}(t)=h \int_{-h}^{t} \int_{\theta-h(\theta)}^{\theta} e^{2 k(\theta-t)}\left[\begin{array}{c}
x(\theta-h(\theta)) \\
\dot{x}(s)
\end{array}\right]^{T} \\
& \times\left[\begin{array}{cc}
R_{1}(\alpha) & R_{2}(\alpha) \\
* & R_{3}(\alpha)
\end{array}\right] \\
& \times\left[\begin{array}{c}
x(\theta-h(\theta)) \\
\dot{x}(s)
\end{array}\right] d s d \theta \\
& +\beta h \int_{-\beta h}^{t} \int_{\theta-\beta h(\theta)}^{\theta} e^{2 k(\theta-t)}\left[\begin{array}{c}
x(\theta-\beta h(\theta)) \\
\dot{x}(s)
\end{array}\right]^{T} \\
& \times\left[\begin{array}{cc}
R_{4}(\alpha) & R_{5}(\alpha) \\
* & R_{6}(\alpha)
\end{array}\right] \\
& \times\left[\begin{array}{c}
x(\theta-\beta h(\theta)) \\
\dot{x}(s)
\end{array}\right] d s d \theta \text {. }
\end{aligned}
$$

Calculating the time derivatives of $V_{i}(t), i=1,2,3, \ldots, 7$, along the trajectory of (9), yields

$$
\begin{aligned}
\dot{V}_{1}(t)=2 x^{T}(t) P_{1}(\alpha) \dot{x}(t) & \\
=2 x^{T} P_{1}(\alpha)[ & \left(A(\alpha)+B_{1}(\alpha)+G\right) x(t) \\
& +B_{2}(\alpha) x(t-h(t))-G x(t-\beta h(t)) \\
& +f(t, x(t))+g(t, x(t-h(t))) \\
& +w(t, \dot{x}(t-r(t)))+C(\alpha) \dot{x}(t-r(t)) \\
& -B_{1}(\alpha) \int_{t-h(t)}^{t} \dot{x}(s) d s \\
& \left.-G \int_{t-\beta h(t)}^{t} \dot{x}(s) d s\right] \\
+2 k x^{T}(t) & P_{1}(\alpha) x(t)-2 k V_{1}(t),
\end{aligned}
$$

$$
\begin{aligned}
\dot{V}_{2}(t)= & \dot{x}^{T}(t) P_{2}(\alpha) \dot{x}(t) \\
& -(1-\dot{r}(t)) e^{-2 k r(t)} \dot{x}^{T}(t-r(t)) \\
& \times P_{2}(\alpha) \dot{x}(t-r(t))-2 k V_{2}(t) \\
\leq & \dot{x}^{T}(t) P_{2}(\alpha) \dot{x}(t)-e^{-2 k r} \dot{x}^{T}(t-r(t)) \\
& \times P_{2}(\alpha) \dot{x}(t-r(t)) \\
& +r_{d} \dot{x}^{T}(t-r(t)) P_{2}(\alpha) \dot{x}(t-r(t)) \\
& -2 k V_{2}(t) .
\end{aligned}
$$

The time derivative of $V_{3}(t)$ is

$$
\begin{aligned}
\dot{V}_{3}(t)= & x^{T}(t) P_{3}(\alpha) x(t)-(1-\dot{h}(t)) e^{-2 k h(t)} x^{T}(t-h(t)) \\
& \times P_{3}(\alpha) x(t-h(t))+x^{T}(t) P_{4}(\alpha) x(t) \\
& -(1-\beta \dot{h}(t)) e^{-2 k \beta h(t)} x^{T}(t-\beta h(t)) \\
& \times P_{4}(\alpha) x(t-\beta h(t))-2 k V_{3}(t) \\
\leq & x^{T}(t) P_{3}(\alpha) x(t)-e^{-2 k h} x^{T}(t-h(t)) \\
& \times P_{3}(\alpha) x(t-h(t))+h_{d} e^{-2 k h} x^{T}(t-h(t)) \\
& \times P_{3}(\alpha) x(t-h(t))+x^{T}(t) P_{4}(\alpha) x(t) \\
& -e^{-2 \alpha \beta h} x^{T}(t-\beta h(t)) P_{4}(\alpha) x(t-\beta h(t)) \\
& +\beta h_{d} e^{-2 k \beta h} x^{T}(t-\beta h(t)) P_{4}(\alpha) x(t-\beta h(t)) \\
& -2 k V_{3}(t) .
\end{aligned}
$$

Obviously, for any scalar $s \in[t-h, t]$, we get $e^{-2 k h} \leq$ $e^{-2 k(s-t)} \leq 1$ and for any scalar $s \in[t-\beta h, t]$, we obtain $e^{-2 \beta k h} \leq e^{-2 \beta k(s-t)} \leq 1$. Together with Lemma 4 , we obtain

$$
\begin{aligned}
\dot{V}_{4}(t)= & h^{2} \dot{x}^{T}(t) P_{5}(\alpha) \dot{x}(t) \\
& -h \int_{-h}^{0} e^{2 k s} \dot{x}^{T}(t+s) P_{5}(\alpha) \dot{x}(t+s) d s \\
& +\beta^{2} h^{2} \dot{x}^{T}(t) P_{6}(\alpha) \dot{x}(t) \\
& -\beta h \int_{-\beta h}^{0} e^{2 \beta k s} \dot{x}^{T}(t+s) P_{6}(\alpha) \dot{x}(t+s) d s \\
& -2 k V_{4}(t)
\end{aligned}
$$




$$
\begin{aligned}
& \leq h^{2} \dot{x}^{T}(t) P_{5}(\alpha) \dot{x}(t) \\
& -h \int_{t-h}^{t} e^{2 k(s-t)} \dot{x}^{T}(s) P_{5}(\alpha) \dot{x}(s) d s \\
& +\beta^{2} h^{2} \dot{x}^{T}(t) P_{6}(\alpha) \dot{x}(t) \\
& -\beta h \int_{t-\beta h}^{t} e^{2 \beta k(s-t)} \dot{x}^{T}(s) P_{6}(\alpha) \dot{x}(s) d s \\
& -2 k V_{4}(t) \\
& \leq h^{2} \dot{x}^{T}(t) P_{5}(\alpha) \dot{x}(t) \\
& -h e^{-2 k h} \int_{t-h}^{t} \dot{x}^{T}(s) P_{5}(\alpha) \dot{x}(s) d s \\
& +\beta^{2} h^{2} \dot{x}^{T}(t) P_{6}(\alpha) \dot{x}(t) \\
& -\beta h e^{-2 \beta k h} \int_{t-\beta h}^{t} \dot{x}^{T}(s) P_{6}(\alpha) \dot{x}(s) d s \\
& -2 k V_{4}(t) \\
& \dot{V}_{5}(t) \leq h^{2} \dot{x}^{T}(t) P_{7}(\alpha) \dot{x}(t) \\
& -h e^{-2 k h} \int_{t-h}^{t} \dot{x}^{T}(s) P_{7}(\alpha) \dot{x}(s) d s \\
& +\beta^{2} h^{2} \dot{x}^{T}(t) P_{8}(\alpha) \dot{x}(t) \\
& -\beta h e^{-2 \beta k h} \int_{t-\beta h}^{t} \dot{x}^{T}(s) P_{8}(\alpha) \dot{x}(s) d s \\
& -2 k V_{5}(t) \\
& \leq h^{2} \dot{x}^{T}(t) P_{7}(\alpha) \dot{x}(t) \\
& -e^{-2 k h} \int_{t-h}^{t} \dot{x}^{T}(s) d s P_{7}(\alpha) \int_{t-h}^{t} \dot{x}(s) d s \\
& +\beta^{2} h^{2} \dot{x}^{T}(t) P_{8}(\alpha) \dot{x}(t) \\
& -e^{-2 \beta k h} \int_{t-\beta h}^{t} \dot{x}^{T}(s) d s P_{8}(\alpha) \int_{t-\beta h}^{t} \dot{x}(s) d s \\
& -2 k V_{5}(t) \\
& \leq h^{2} \dot{x}^{T}(t) P_{7}(\alpha) \dot{x}(t) \\
& -e^{-2 k h} \int_{t-h(t)}^{t} \dot{x}^{T}(s) d s P_{7}(\alpha) \int_{t-h(t)}^{t} \dot{x}(s) d s \\
& +\beta^{2} h^{2} \dot{x}^{T}(t) P_{8}(\alpha) \dot{x}(t) \\
& -e^{-2 \beta k h} \int_{t-\beta h(t)}^{t} \dot{x}^{T}(s) d s P_{8}(\alpha) \int_{t-\beta h(t)}^{t} \dot{x}(s) d s \\
& -2 \alpha V_{5}(t), \\
& \dot{V}_{6}(t) \leq h^{2} \dot{x}^{T}(t) P_{9}(\alpha) \dot{x}(t) \\
& -e^{-2 k h} \int_{t-h(t)}^{t} \dot{x}^{T}(s) d s P_{9}(\alpha) \int_{t-h(t)}^{t} \dot{x}(s) d s
\end{aligned}
$$

$$
\begin{aligned}
& +\beta^{2} h^{2} \dot{x}^{T}(t) P_{10}(\alpha) \dot{x}(t) \\
& -e^{-2 \beta k h} \int_{t-\beta h(t)}^{t} \dot{x}^{T}(s) d s P_{10}(\alpha) \int_{t-\beta h(t)}^{t} \dot{x}(s) d s \\
& -2 k V_{6}(t) \\
= & h^{2} \dot{x}^{T}(t) P_{9}(\alpha) \dot{x}(t) \\
& -e^{-2 k h}\left[x^{T}(t)-x^{T}(t-h(t))\right] \\
& \times P_{9}(\alpha)[x(t)-x(t-h(t))] \\
& +\beta h^{2} \dot{x}^{T}(t) P_{10}(\alpha) \dot{x}(t) \\
& -e^{-2 \beta k h}\left[x^{T}(t)-x^{T}(t-\beta h(t))\right] \\
& \times P_{10}(\alpha)[x(t)-x(t-\beta h(t))]-2 k V_{6}(t) .
\end{aligned}
$$

Taking the time derivative of $V_{7}(t)$, we obtain

$$
\begin{aligned}
& \dot{V}_{7}(t)=h h(t) x^{T}(t-h(t)) R_{1}(\alpha) x(t-h(t)) \\
& +2 h x^{T}(t-h(t)) R_{2}(\alpha) x(t) \\
& -2 h x^{T}(t-h(t)) R_{2}(\alpha) x(t-h(t)) \\
& +h \int_{t-h}^{t} \dot{x}^{T}(s) R_{3}(\alpha) \dot{x}(s) d s \\
& +\beta^{2} h h(t) x^{T}(t-\beta h(t)) R_{4}(\alpha) x(t-\beta h(t)) \\
& +2 \beta h x^{T}(t-h(t)) R_{5}(\alpha) x(t) \\
& -2 \beta h x^{T}(t-\beta h(t)) R_{5}(\alpha) x(t-\beta h(t)) \\
& +\beta h \int_{t-\beta h}^{t} \dot{x}^{T}(s) R_{6}(\alpha) \dot{x}(s) d s-2 k V_{7}(t) \\
& \leq h^{2} x^{T}(t-h(t)) R_{1}(\alpha) x(t-h(t)) \\
& +2 h x^{T}(t-h(t)) R_{2}(\alpha) x(t) \\
& -2 h x^{T}(t-h(t)) R_{2}(\alpha) x(t-h(t)) \\
& +h \int_{t-h}^{t} \dot{x}^{T}(s) R_{3}(\alpha) \dot{x}(s) d s \\
& +\beta^{2} h^{2} x^{T}(t-\beta h(t)) R_{4}(\alpha) x(t-\beta h(t)) \\
& +2 \beta h x^{T}(t-h(t)) R_{5}(\alpha) x(t) \\
& -2 \beta h x^{T}(t-\beta h(t)) R_{5}(\alpha) x(t-\beta h(t)) \\
& +\beta h \int_{t-\beta h}^{t} \dot{x}^{T}(s) R_{6}(\alpha) \dot{x}(s) d s-2 k V_{7}(t) \text {. }
\end{aligned}
$$


By Lemma 5 and the integral term of the right hand side of $\dot{V}_{4}(t)$ and $\dot{V}_{7}(t)$, we obtain

$$
\begin{aligned}
& -h \int_{t-h}^{t} \dot{x}^{T}(s)\left[e^{-2 k h} P_{5}(\alpha)-R_{3}(\alpha)\right] \dot{x}(s) d s \\
& -\beta h \int_{t-\beta h}^{t} \dot{x}^{T}(s)\left[e^{-2 \beta k h} P_{6}(\alpha)-R_{6}(\alpha)\right] \dot{x}(s) d s \\
& \leq h\left[\begin{array}{c}
x(t) \\
x t-h(t)
\end{array}\right]^{T} \\
& \times\left[\begin{array}{cc}
M_{1}^{T}(\alpha)+M_{1}(\alpha) & -M_{1}^{T}(\alpha)+M_{2}(\alpha) \\
* & -M_{2}^{T}(\alpha)-M_{2}(\alpha)
\end{array}\right] \\
& \times\left[\begin{array}{c}
x(t) \\
x t-h(t)
\end{array}\right] \\
& +h^{2}\left[\begin{array}{c}
x(t) \\
x t-h(t)
\end{array}\right]^{T}\left[\begin{array}{cc}
M_{3}(\alpha) & M_{4}(\alpha) \\
* & M_{5}(\alpha)
\end{array}\right] \\
& \times\left[\begin{array}{c}
x(t) \\
x t-h(t)
\end{array}\right]+\beta h\left[\begin{array}{c}
x(t) \\
x t-\beta h(t)
\end{array}\right]^{T} \\
& \times\left[\begin{array}{cc}
M_{6}^{T}(\alpha)+M_{6}(\alpha) & -M_{6}^{T}(\alpha)+M_{7}(\alpha) \\
* & -M_{7}^{T}(\alpha)-M_{7}(\alpha)
\end{array}\right] \\
& \times\left[\begin{array}{c}
x(t) \\
x t-\beta h(t)
\end{array}\right] \\
& +\beta^{2} h^{2}\left[\begin{array}{c}
x(t) \\
x t-\beta h(t)
\end{array}\right]^{T}\left[\begin{array}{cc}
M_{8}(\alpha) & M_{9}(\alpha) \\
* & M_{10}(\alpha)
\end{array}\right] \\
& \times\left[\begin{array}{c}
x(t) \\
x t-\beta h(t)
\end{array}\right] .
\end{aligned}
$$

From the Leibniz-Newton formula, the following equations are true for any parameter-dependent real matrices $N_{i}(\alpha), O_{i}(\alpha), i=1,2, \ldots, 10$ with appropriate dimensions:

$$
\begin{aligned}
& 2\left[x^{T}(t) N_{1}^{T}(\alpha)+x^{T}(t-h(t)) N_{2}^{T}(\alpha)\right. \\
& \quad+x^{T}(t-\beta h(t)) N_{3}^{T}(\alpha)+\int_{t-h(t)}^{t} \dot{x}^{T}(s) d s N_{4}^{T}(\alpha) \\
& \quad+\int_{t-\beta h(t)}^{t} \dot{x}^{T}(s) d s N_{5}^{T}(\alpha)+\dot{x}^{T}(t) N_{6}^{T}(\alpha) \\
& \quad+\dot{x}^{T}(t-r(t)) N_{7}^{T}(\alpha)+f^{T}(t, x(t)) N_{8}^{T}(\alpha) \\
& \left.\quad+g^{T}(t, x(t-h(t))) N_{9}^{T}(\alpha)+w^{T}(t, \dot{x}(t-r(t))) N_{10}^{T}(\alpha)\right] \\
& \quad \times\left[x(t)-x(t-h(t))-\int_{t-h(t)}^{t} \dot{x}(s) d s\right]=0,
\end{aligned}
$$

$$
\begin{aligned}
& 2\left[x^{T}(t) O_{1}^{T}(\alpha)+x^{T}(t-h(t)) O_{2}^{T}(\alpha)\right. \\
& \quad+x^{T}(t-\beta h(t)) O_{3}^{T}(\alpha)+\int_{t-h(t)}^{t} \dot{x}^{T}(s) d s O_{4}^{T}(\alpha) \\
& \quad+\int_{t-\beta h(t)}^{t} \dot{x}^{T}(s) d s O_{5}^{T}(\alpha)+\dot{x}^{T}(t) O_{6}^{T}(\alpha) \\
& \quad+\dot{x}^{T}(t-r(t)) O_{7}^{T}(\alpha)+f^{T}(t, x(t)) O_{8}^{T}(\alpha) \\
& \left.\quad+g^{T}(t, x(t-h(t))) O_{9}^{T}(\alpha)+w^{T}(t, \dot{x}(t-r(t))) O_{10}^{T}(\alpha)\right] \\
& \quad \times\left[x(t)-x(t-\beta h(t))-\int_{t-\beta h(t)}^{t} \dot{x}(s) d s\right]=0 .
\end{aligned}
$$

From the utilization of zero equation, the following equation is true for any parameter-dependent real matrices $W_{i}, i=1,2, \ldots, 10$ with appropriate dimensions:

$$
\begin{aligned}
2\left[x^{T}(t) W_{1}^{T}(\alpha)+x^{T}(t-h(t)) W_{2}^{T}(\alpha)\right. & \\
+ & x^{T}(t-\beta h(t)) W_{3}^{T}(\alpha)+\int_{t-h(t)}^{t} \dot{x}^{T}(s) d s W_{4}^{T}(\alpha) \\
+ & \int_{t-\beta h(t)}^{t} \dot{x}^{T}(s) d s W_{5}^{T}(\alpha)+\dot{x}^{T}(t) W_{6}^{T}(\alpha) \\
+ & \dot{x}^{T}(t-r(t)) W_{7}^{T}(\alpha)+f^{T}(t, x(t)) W_{8}(\alpha) \\
+ & g^{T}(t, x(t-h(t))) W_{9}^{T}(\alpha) \\
+ & \left.w^{T}(t, \dot{x}(t-r(t))) W_{10}^{T}(\alpha)\right] \\
\times & {\left[\left(A(\alpha)+B_{1}(\alpha)\right) x(t)+B_{2}(\alpha) x(t-h(t))\right.} \\
& +C(\alpha) \dot{x}(t-r(t))+f(t, x(t))+g(t, x(t-h(t))) \\
& \left.+w(t, \dot{x}(t-r(t)))-B_{1}(\alpha) \int_{t-h(t)}^{t} \dot{x}(s) d s-\dot{x}(t)\right]
\end{aligned}
$$$$
=0 .
$$

From (5), we obtain, for any positive real constants $\epsilon_{1}, \epsilon_{2}$, and $\epsilon_{3}$,

$$
\begin{aligned}
0 \leq & \epsilon_{1} \eta^{2} x^{T}(t) x(t)-\epsilon_{1} f^{T}(t, x(t)) f(t, x(t)), \\
0 \leq & \epsilon_{2} \rho^{2} x^{T}(t-h(t)) x(t-h(t)) \\
& -\epsilon_{2} g^{T}(t, x(t-h(t))) g(t, x(t-h(t))), \\
0 \leq & \epsilon_{3} \gamma^{2} \dot{x}^{T}(t-r(t)) \dot{x}(t-r(t)) \\
& \quad \epsilon_{3} w^{T}(t, \dot{x}(t-r(t))) w(t, \dot{x}(t-r(t))) .
\end{aligned}
$$


According to (29)-(36), it is straightforward to see that

$$
\dot{V}(t) \leq \zeta^{T}(t) \sum_{i=1}^{N} \sum_{j=1}^{N} \alpha_{i} \alpha_{j} \sum_{i, j} \zeta(t)-2 k V(t),
$$

where $\zeta^{T}(t)=\left[x^{T}(t), x^{T}(t-h(t)), x^{T}(t-\beta h(t)), \int_{t-h(t)}^{t} \dot{x}^{T}(s) d s\right.$, $\int_{t-\beta h(t)}^{t} \dot{x}^{T}(s) d s, \dot{x}^{T}(t), \dot{x}^{T}(t-r(t)), f^{T}(t, x(t)), g^{T}(t, x(t-$ $h(t))), w^{T}(t, \dot{x}(t-r(t))]$ and $\sum_{i, j}$ is defined in (23). From the fact that $\sum_{i=1}^{N} \alpha_{i}=1$,

$$
\begin{gathered}
\sum_{i=1}^{N} \alpha_{i} A_{i} \sum_{i=1}^{N} \alpha_{i} B_{i}=\sum_{i=1}^{N} \alpha_{i}^{2} A_{i} B_{i}+\sum_{i=1}^{N-1} \sum_{j=i+1}^{N} \alpha_{i} \alpha_{j}\left[A_{i} B_{j}+A_{j} B_{i}\right] \\
(N-1) \sum_{i=1}^{N} \alpha_{i}^{2}-2 \sum_{i=1}^{N-1} \sum_{j=i+1}^{N} \alpha_{i} \alpha_{j}=\sum_{i=1}^{N-1} \sum_{j=i+1}^{N}\left[\alpha_{i}-\alpha_{j}\right]^{2} \geq 0
\end{gathered}
$$

It is true that if conditions (25) hold, then

$$
\dot{V}(t)+2 k V(t) \leq 0, \quad \forall t \in R^{+},
$$

which gives

$$
V(t) \leq V(0) e^{-2 k t}, \quad \forall t \in R^{+} .
$$

From (40), it is easy to see that

$$
\begin{gathered}
\lambda_{\min }\left(P_{1}(\alpha)\right)\|x(t)\|^{2} \leq V(t) \leq V(0) e^{-2 k t}, \\
V(0)=\sum_{i=1}^{7} V_{i}(0),
\end{gathered}
$$

where

$$
\begin{gathered}
V_{1}(0)=x^{T}(0) P_{1}(\alpha) x(0), \\
V_{2}(0)=\int_{-r(0)}^{0} e^{2 k s} \dot{x}^{T}(s) P_{2}(\alpha) \dot{x}(s) d s, \\
V_{3}(0)=\int_{-h(0)}^{0} e^{2 k s} x^{T}(s) P_{3}(\alpha) x(s) d s \\
+\int_{-\beta h(0)}^{0} e^{2 k s} x^{T}(s) P_{4}(\alpha) x(s) d s, \\
V_{4}(0)=h \int_{-h}^{0} \int_{\theta}^{0} e^{2 k s} \dot{x}^{T}(s) P_{5}(\alpha) \dot{x}(s) d s d \theta \\
+\beta h \int_{-\beta h}^{0} \int_{\theta}^{0} e^{2 k s} \dot{x}^{T}(s) P_{6}(\alpha) \dot{x}(s) d s d \theta, \\
V_{5}(0)=h \int_{-h}^{0} \int_{\theta}^{0} e^{2 k s} \dot{x}^{T}(s) P_{7}(\alpha) \dot{x}(s) d s d \theta \\
+\beta h \int_{-\beta h}^{0} \int_{\theta}^{0} e^{2 k s} \dot{x}^{T}(s) P_{8}(\alpha) \dot{x}(s) d s d \theta, \\
V_{6}(0)=h \int_{-h}^{0} \int_{\theta}^{t} e^{2 k s} \dot{x}^{T}(s) P_{9}(\alpha) \dot{x}(s) d s d \theta \\
+\beta h \int_{\theta}^{0} e^{2 k s} \dot{x}^{T}(s) P_{10}(\alpha) \dot{x}(s) d s d \theta,
\end{gathered}
$$

$$
\begin{aligned}
& V_{7}(0)=h \int_{-h}^{0} \int_{\theta-h(\theta)}^{\theta} e^{2 k \theta}\left[\begin{array}{c}
x(\theta-h(\theta)) \\
\dot{x}(s)
\end{array}\right]^{T} \\
& \times\left[\begin{array}{cc}
R_{1}(\alpha) & R_{2}(\alpha) \\
* & R_{3}(\alpha)
\end{array}\right] \\
& \times\left[\begin{array}{c}
x(\theta-h(\theta)) \\
\dot{x}(s)
\end{array}\right] d s d \theta \\
& +\beta h \int_{-\beta h}^{0} \int_{\theta-\beta h(\theta)}^{\theta} e^{2 k \theta}\left[\begin{array}{c}
x(\theta-\beta h(\theta)) \\
\dot{x}(s)
\end{array}\right]^{T} \\
& \times\left[\begin{array}{cc}
R_{4}(\alpha) & R_{5}(\alpha) \\
* & R_{6}(\alpha)
\end{array}\right] \\
& \times\left[\begin{array}{c}
x(\theta-\beta h(\theta)) \\
\dot{x}(s)
\end{array}\right] d s d \theta .
\end{aligned}
$$

From (41), we conclude that

$\lambda_{\min }\left(P_{1}(\alpha)\right)\|x(t)\|^{2} \leq V(0) e^{-2 k t} \leq L \max [\|\phi\|,\|\varphi\|]^{2} e^{-2 k t}$,

where $L=\lambda_{\text {max }}\left(P_{1}(\alpha)\right)+r \lambda_{\text {max }}\left(P_{2}(\alpha)\right)+h \lambda_{\text {max }}\left(P_{3}(\alpha)\right)+$ $\beta h \lambda_{\max }\left(P_{4}(\alpha)\right)+h^{3} \lambda_{\max }\left(P_{5}(\alpha)+P_{7}(\alpha)+P_{9}(\alpha)\right)+(\beta h)^{3}$ $\lambda_{\max }\left(P_{6}(\alpha)+P_{8}(\alpha)+P_{10}(\alpha)\right)+h^{3} \lambda_{\max }\left(\left[\begin{array}{rr}R_{1}(\alpha) & R_{2}(\alpha) \\ * & R_{3}(\alpha)\end{array}\right]\right)+(\beta h)^{3}$ $\lambda_{\max }\left(\left[\begin{array}{cc}R_{4}(\alpha) & R_{5}(\alpha) \\ * & R_{6}(\alpha)\end{array}\right]\right)$. From (44), this means that the system (1) is robustly exponentially stable. The proof of the theorem is complete.

Next, we consider the following system:

$$
\begin{aligned}
& \dot{x}(t)= A(\alpha) x(t)+B(\alpha) x(t-h(t))+C(\alpha) \dot{x}(t-r(t)) \\
&+f(t, x(t))+g(t, x(t-h(t))), \quad t>0 ; \\
& x(t)=\phi(t), \quad \dot{x}(t)=\psi(t), \quad t \in[-b, 0] .
\end{aligned}
$$

We introduce the following notations for later use:

$$
\prod_{i, j}=\left[\begin{array}{ccccccccc}
\Sigma_{i, j}^{1,1} & \Sigma_{i, j}^{1,2} & \Sigma_{i, j}^{1,3} & \Sigma_{i, j}^{1,4} & \Sigma_{i, j}^{1,5} & \Sigma_{i, j}^{1,6} & \Sigma_{i, j}^{1,7} & \Sigma_{i, j}^{1,8} & \Sigma_{i, j}^{1,9} \\
* & \Sigma_{i, j}^{2,2} & \Sigma_{i, j}^{2,3} & \Sigma_{i, j}^{2,4} & \Sigma_{i, j}^{2,5} & \Sigma_{i, j}^{2,6} & \Sigma_{i, j}^{2,7} & \Sigma_{i, j}^{2,8} & \Sigma_{i, j}^{2,9} \\
* & * & \Sigma_{i}^{3,3} & \Sigma_{i, j}^{3,4} & \Sigma_{i}^{3,5} & \Sigma_{i}^{3,6} & \Sigma_{i, j}^{3,7} & \Sigma_{i}^{3,8} & \Sigma_{i}^{3,9} \\
* & * & * & \Sigma_{i, j}^{4,4} & \Sigma_{i, j}^{4,5} & \Sigma_{i, j}^{4,6} & \Sigma_{i, j}^{4,7} & \Sigma_{i}^{4,8} & \Sigma_{i}^{4,9} \\
* & * & * & * & \Sigma_{i}^{5,5} & \Sigma_{i}^{5,6} & \Sigma_{i, j}^{5,7} & \Sigma_{i}^{5,8} & \Sigma_{i}^{5,9} \\
* & * & * & * & * & \Sigma_{i}^{6,6} & \Sigma_{i, j}^{6,7} & \Sigma_{i}^{6,8} & \Sigma_{i}^{6,9} \\
* & * & * & * & * & * & \widehat{\Sigma}_{i, j}^{7,7} & \Sigma_{i, j}^{7,8} & \Sigma_{i, j}^{7,9} \\
* & * & * & * & * & * & * & \Sigma_{i}^{8,8} & \Sigma_{i}^{8,9} \\
* & * & * & * & * & * & * & * & \Sigma_{i}^{9,9}
\end{array}\right] .
$$

Corollary 8. For $\left\|C_{i}\right\|<1, i=1,2, \ldots, N$ and given positive real constants $h, h_{d}, r, r_{d}, \eta, \rho$, and $\beta$, system (45) is robustly exponentially stable with a decay rate $k$, if there exist symmetric positive definite matrices $P_{i}^{j}$, any appropriate dimensional matrices $R_{i}^{p}, M_{i}^{l}, N_{i}^{j}, O_{i}^{j}, W_{i}^{j}, p=1,2, \ldots 6$, $l=1,2, \ldots, 10, j=1,2, \ldots, 9, i=1,2, \ldots, N$, and positive 
real constants $\epsilon_{1}, \epsilon_{2}$ such that the following symmetric linear matrix inequalities hold:

$$
\begin{gathered}
{\left[\begin{array}{cc}
R_{i}^{1} & R_{i}^{2} \\
* & R_{i}^{3}
\end{array}\right]>0, \quad i=1,2, \ldots, N,} \\
{\left[\begin{array}{cc}
R_{i}^{4} & R_{i}^{5} \\
* & R_{i}^{6}
\end{array}\right]>0, \quad i=1,2, \ldots, N,} \\
{\left[\begin{array}{ccc}
e^{-2 k h} P_{i}^{3}-R_{i}^{3} & M_{i}^{1} & M_{i}^{2} \\
* & M_{i}^{3} & M_{i}^{4} \\
* & * & M_{i}^{5}
\end{array}\right] \geq 0, \quad i=1,2, \ldots, N,} \\
{\left[\begin{array}{ccc}
e^{-2 \beta k h} P_{i}^{6}-R_{i}^{6} & M_{i}^{6} & M_{i}^{7} \\
* & M_{i}^{8} & M_{i}^{9} \\
* & * & M_{i}^{10}
\end{array}\right] \geq 0, \quad i=1,2, \ldots, N,} \\
\prod_{i, j}+\prod_{j, i}<\frac{2}{(N-1)} I, \quad i=1,2, \ldots, N-1, \\
\quad j=i+1, i+2, \ldots, N .
\end{gathered}
$$

Moreover, the solution $x(t, \phi, \psi)$ satisfies the inequality

$\|x(t, \phi, \psi)\| \leq \sqrt{\frac{L}{\lambda_{\min }\left(P_{1}(\alpha)\right)}} \max [\|\phi\|,\|\psi\|] e^{-k t}$,

$\forall t \in R^{+}$,

where $L=\lambda_{\max }\left(P_{1}(\alpha)\right)+r \lambda_{\max }\left(P_{2}(\alpha)\right)+h \lambda_{\max }\left(P_{3}(\alpha)\right)+$ $\beta h \lambda_{\max }\left(P_{4}(\alpha)\right)+h^{3} \lambda_{\max }\left(P_{5}(\alpha)+P_{7}(\alpha)+P_{9}(\alpha)\right)+$ $(\beta h)^{3} \lambda_{\max }\left(P_{6}(\alpha)+P_{8}(\alpha)+P_{10}(\alpha)\right)+h^{3} \lambda_{\max }\left(\left[\begin{array}{cc}R_{1}(\alpha) & R_{2}(\alpha) \\ * & R_{3}(\alpha)\end{array}\right]\right)+$ $(\beta h)^{3} \lambda_{\max }\left(\left[\begin{array}{cc}R_{4}(\alpha) & R_{5}(\alpha) \\ * & R_{6}(\alpha)\end{array}\right]\right)$.

3.2. Exponential Stability Criteria. In this section, we study the exponential stability criteria for neutral systems with time-varying delays by using the combination of linear matrix inequality (LMI) technique and Lyapunov theory method. We introduce the following notations for later use:

$$
\Sigma=\left[\begin{array}{cccccccccc}
\Sigma_{11} & \Sigma_{12} & \Sigma_{13} & \Sigma_{14} & \Sigma_{15} & \Sigma_{16} & \Sigma_{17} & \Sigma_{18} & \Sigma_{19} & \Sigma_{1,10} \\
* & \Sigma_{22} & \Sigma_{23} & \Sigma_{24} & \Sigma_{25} & \Sigma_{26} & \Sigma_{27} & \Sigma_{28} & \Sigma_{29} & \Sigma_{2,10} \\
* & * & \Sigma_{33} & \Sigma_{34} & \Sigma_{35} & \Sigma_{36} & \Sigma_{37} & \Sigma_{38} & \Sigma_{39} & \Sigma_{3,10} \\
* & * & * & \Sigma_{44} & \Sigma_{45} & \Sigma_{46} & \Sigma_{47} & \Sigma_{48} & \Sigma_{49} & \Sigma_{4,10} \\
* & * & * & * & \Sigma_{55} & \Sigma_{56} & \Sigma_{57} & \Sigma_{58} & \Sigma_{59} & \Sigma_{5,10} \\
* & * & * & * & * & \Sigma_{66} & \Sigma_{67} & \Sigma_{68} & \Sigma_{69} & \Sigma_{6,10} \\
* & * & * & * & * & * & \Sigma_{77} & \Sigma_{78} & \Sigma_{79} & \Sigma_{7,10} \\
* & * & * & * & * & * & * & \Sigma_{88} & \Sigma_{89} & \Sigma_{8,10} \\
* & * & * & * & * & * & * & * & \Sigma_{99} & \Sigma_{9,10} \\
* & * & * & * & * & * & * & * & * & \Sigma_{10,10}
\end{array}\right],
$$

where

$$
\begin{aligned}
& \Sigma_{1,1}=A^{T} P_{1}+B_{1}^{T} P_{1}+Z_{1}^{T}+P_{1} A+P_{1} B_{1}+Z_{1}+P_{3} \\
& +P_{4}+h M_{1}^{T}+h M_{1}-e^{-2 k h} P_{9}-e^{-2 k \beta h} P_{10} \\
& +h^{2} M_{3}+\beta h M_{6}+\beta h M_{6}^{T}+\beta^{2} h^{2} M_{8} \\
& +N_{1}^{T}+N_{1}+O_{1}^{T}+O_{1}+A^{T} W_{1}+W_{1}^{T} A \\
& +B_{1}^{T} W_{1}+W_{1}^{T} B_{1}+\epsilon_{1} \eta^{2} I+2 k P_{1}, \\
& \Sigma_{1,2}=P_{1} B_{2}-h M_{1}^{T}+h M_{2}+h^{2} M_{4}+e^{-2 k h} P_{9} \\
& +h R_{2}-N_{1}^{T}+N_{2}+O_{2}+W_{1}^{T} B_{2} \\
& +A^{T} W_{2}+B_{1}^{T} W_{2} \\
& \Sigma_{1,3}=-Z_{1}-\beta h M_{6}^{T}+\beta h M_{7}+\beta h^{2} M_{9}+\beta h R_{5} \\
& +e^{-2 k \beta h} P_{10}+N_{3}-O_{1}^{T}+O_{3} \\
& +A^{T} W_{3}+B_{1}^{T} W_{3} \\
& \Sigma_{1,4}=-P_{1} B_{1}-N_{1}^{T}+N_{4}+O_{4} \\
& -W_{1}^{T} B_{1}+A^{T} W_{4}+B_{1}^{T} W_{4}, \\
& \Sigma_{1,5}=-Z_{1}+N_{5}-O_{1}^{T}+O_{5}+A^{T} W_{5}+B_{1}^{T} W_{5}, \\
& \Sigma_{1,6}=N_{6}+O_{6}-W_{1}^{T}+A^{T} W_{6}+B_{1}^{T} W_{6}, \\
& \Sigma_{1,7}=P_{1} C+N_{7}+O_{7}+W_{1}^{T} C+A^{T} W_{7}+B_{1}^{T} W_{7} \text {, } \\
& \Sigma_{1,8}=P_{1}+N_{8}+O_{8}+W_{1}^{T}+A^{T} W_{8}+B_{1}^{T} W_{8} \text {, } \\
& \Sigma_{1,9}=P_{1}+N_{9}+O_{9}+W_{1}^{T}+A^{T} W_{9}+B_{1}^{T} W_{9}, \\
& \Sigma_{1,10}=P_{1}+N_{10}+O_{10}+W_{1}^{T}+A^{T} W_{10}+B_{1}^{T} W_{10}, \\
& \Sigma_{2,2}=-\left(1-h_{d}\right) e^{-2 k h} P_{3}-h M_{2}^{T}-h M_{2} \\
& +h^{2} M_{5}+h^{2} R_{1}-2 h R_{2}-e^{-2 k h} P_{9} \\
& +\epsilon_{2} \rho^{2} I-N_{2}^{T}-N_{2}+W_{2}^{T} B_{2}+B_{2}^{T} W_{2}, \\
& \Sigma_{2,3}=-N_{3}-O_{2}^{T}+B_{2}^{T} W_{3}, \\
& \Sigma_{2,4}=-N_{2}^{T}-N_{4}-W_{2}^{T} B_{1}+B_{2}^{T} W_{4}, \\
& \Sigma_{2,5}=-N_{5}-O_{2}^{T}+B_{2}^{T} W_{5}, \\
& \Sigma_{2,6}=-N_{6}-W_{2}^{T}+B_{2}^{T} W_{6} \text {, } \\
& \Sigma_{2,7}=-N_{7}+W_{2}^{T} C+B_{2}^{T} W_{7} \text {, } \\
& \Sigma_{2,8}=-N_{8}+W_{2}^{T}+B_{2}^{T} W_{8}, \\
& \Sigma_{2,9}=-N_{9}^{T}-W_{2}^{T}+B_{2}^{T} W_{9}, \\
& \Sigma_{2,10}=-N_{10}+W_{2}^{T}+B_{2}^{T} W_{10} \text {, }
\end{aligned}
$$




$$
\begin{aligned}
& \Sigma_{3,3}=-\left(1-\beta h_{d}\right) e^{-2 \beta k h} P_{4}-e^{-2 \beta k h} P_{10}+\beta^{2} h^{2} R_{4} \\
& -2 \beta h R_{5}-\beta h M_{7}^{T}-\beta h M_{7}+\beta^{2} h^{2} M_{10} \\
& -\mathrm{O}_{3}^{T}-\mathrm{O}_{3} \\
& \Sigma_{3,4}=-N_{3}^{T}-O_{4}-W_{3}^{T} B_{1}, \\
& \Sigma_{3,5}=-O_{3}^{T}-O_{5} \text {, } \\
& \Sigma_{3,6}=-O_{9}+W_{3}^{T}, \quad \Sigma_{3,7}=-O_{7}+W_{3}^{T} C, \\
& \Sigma_{3,8}=-O_{8}+W_{3}^{T}, \quad \Sigma_{3,9}=-O_{9}+W_{3}^{T}, \\
& \Sigma_{3,10}=-O_{10}+W_{3}^{T}, \\
& \Sigma_{4,4}=-e^{2 k h} P_{7}-N_{4}^{T}-N_{4}+W_{4}^{T} B_{1}-B_{1}^{T} W_{4}, \\
& \Sigma_{4,5}=-N_{5}-O_{4}^{T}-B_{1}^{T} W_{5}, \\
& \Sigma_{4,6}=-N_{6}-W_{4}^{T}-B_{1}^{T} W_{6} \text {, } \\
& \Sigma_{4,7}=-N_{7}+W_{4}^{T} C-B_{1}^{T} W_{7}, \\
& \Sigma_{4,8}=-O_{8}+W_{3}^{T}, \\
& \Sigma_{4,9}=-O_{9}+W_{3}^{T}, \quad \Sigma_{4,10}=-O_{10}+W_{3}^{T} \text {, } \\
& \Sigma_{5,5}=-e^{2 \beta k h} P_{8}-O_{5}^{T}-O_{5} \text {, } \\
& \Sigma_{5,6}=-\mathrm{O}_{6}-W_{5}^{T}, \\
& \Sigma_{5,7}=-O_{7}+W_{5}^{T} C, \quad \Sigma_{5,8}=-O_{8}+W_{5}^{T} \text {, } \\
& \Sigma_{5,9}=-O_{9}+W_{5}^{T}, \\
& \Sigma_{5,10}=-O_{10}+W_{5}^{T} \text {, } \\
& \Sigma_{6,6}=P_{2}+h^{2} P_{5}+\beta^{2} h^{2} P_{6}+h^{2} P_{7}+\beta^{2} h^{2} P_{8} \\
& +h^{2} P_{9}+\beta^{2} h^{2} P_{10}-W_{6}^{T}-W_{6}, \\
& \Sigma_{6,7}=W_{6}^{T} C-W_{7}, \quad \Sigma_{6,8}=W_{6}^{T}-W_{8}, \\
& \Sigma_{6,9}=W_{6}^{T}-W_{9}, \quad \Sigma_{6,10}=W_{6}^{T}-W_{10} \text {, } \\
& \Sigma_{7,7}=-\left(1-r_{d}\right) e^{-2 k r} P_{2}+\epsilon_{3} \gamma^{2} I \\
& +W_{7}^{T} C+C^{T} W_{7} \text {, } \\
& \Sigma_{7,8}=W_{7}^{T}-C^{T} W_{8}, \quad \Sigma_{7,9}=W_{7}^{T}-C^{T} W_{9}, \\
& \Sigma_{7,10}=W_{7}^{T}-C^{T} W_{10} \\
& \Sigma_{8,8}=W_{8}^{T}+W_{8}-\epsilon_{1} I \\
& \Sigma_{8,9}=W_{8}^{T}+W_{9}, \quad \Sigma_{8,10}=W_{8}^{T}+W_{10}, \\
& \Sigma_{9,9}=W_{9}^{T}+W_{9}-\epsilon_{2} I, \quad \Sigma_{9,10}=W_{9}^{T}+W_{10} \text {, } \\
& \Sigma_{10,10}=W_{10}^{T}+W_{10}-\epsilon_{3} I, \\
& \widehat{\Sigma_{7,7}}=-\left(1-r_{d}\right) e^{-2 k r} P_{2}+W_{7}^{T} C+C^{T} W_{7} \text {, } \\
& Z_{1}=P_{1} G \text {. }
\end{aligned}
$$

If $A(\alpha)=A, B(\alpha)=B$, and $C(\alpha)=C$, where $A, B, C \in$ $R^{n \times n}$ are real constant matrices, then system (1) reduces to the following system:

$$
\begin{aligned}
\dot{x}(t)= & A x(t)+B x(t-h(t))+C \dot{x}(t-r(t)) \\
& +f(t, x(t))+g(t, x(t-h(t))) \\
& +w(t, \dot{x}(t-r(t))), \quad t>0 ; \\
x(t)= & \phi(t), \quad \dot{x}(t)=\psi(t), \quad t \in[-b, 0] .
\end{aligned}
$$

Corollary 9. For $\|C\|+\gamma<1$ and given positive real constants $h, h_{d}, r, r_{d}, \eta, \rho, \gamma$, and $\beta$, system (51) is exponentially stable with a decay rate $k$, if there exist symmetric positive definite matrices $P_{i}$, any appropriate dimensional matrices $R_{s}, N_{i}, O_{i}, W_{i}, M_{i}$, $G, s=1,2, \ldots 6, i=1,2, \ldots, 10$, and positive real constants $\epsilon_{1}, \epsilon_{2}$, and $\epsilon_{3}$ such that the following symmetric linear matrix inequalities hold:

$$
\begin{gathered}
{\left[\begin{array}{cc}
R_{1} & R_{2} \\
* & R_{3}
\end{array}\right]>0,} \\
{\left[\begin{array}{cc}
R_{4} & R_{5} \\
* & R_{6}
\end{array}\right]>0,} \\
{\left[\begin{array}{ccc}
e^{-2 k h} P_{3}-R_{3} & M_{1} & M_{2} \\
* & M_{3} & M_{4} \\
* & * & M_{5}
\end{array}\right] \geq 0,} \\
{\left[\begin{array}{ccc}
e^{-2 \beta k h} P_{6}-R_{6} & M_{6} & M_{7} \\
* & M_{8} & M_{9} \\
* & * & M_{10}
\end{array}\right] \geq 0,} \\
\sum<0 .
\end{gathered}
$$

Moreover, the solution $x(t, \phi, \psi)$ satisfies the inequality

$\|x(t, \phi, \psi)\| \leq \sqrt{\frac{D}{\lambda_{\min }\left(P_{1}\right)}} \max [\|\phi\|,\|\psi\|] e^{-k t}, \quad \forall t \in R^{+}$,

where $D=\lambda_{\text {max }}\left(P_{1}\right)+r \lambda_{\text {max }}\left(P_{2}\right)+h \lambda_{\text {max }}\left(P_{3}\right)+\beta h \lambda_{\text {max }}\left(P_{4}\right)+$ $h^{3} \lambda_{\text {max }}\left(P_{5}+P_{7}+P_{9}\right)+(\beta h)^{3} \lambda_{\max }\left(P_{6}+P_{8}+P_{10}\right)+$ $h^{3} \lambda_{\max }\left(\left[\begin{array}{cc}R_{1} & R_{2} \\ * & R_{3}\end{array}\right]\right)+(\beta h)^{3} \lambda_{\max }\left(\left[\begin{array}{cc}R_{4} & R_{5} \\ * & R_{6}\end{array}\right]\right)$.

If $A(\alpha)=A, B(\alpha)=B, C(\alpha)=C$, and $w(t, \dot{x}(t-r(t)))=0$, where $A, B, C \in R^{n \times n}$ are real constant matrices, then system (1) reduces to the following system:

$$
\begin{aligned}
\dot{x}(t)= & A x(t)+B x(t-h(t))+C \dot{x}(t-r(t)) \\
& +f(t, x(t))+g(t, x(t-h(t))), \quad t>0 ; \\
x(t)= & \phi(t), \quad \dot{x}(t)=\psi(t), \quad t \in[-b, 0] .
\end{aligned}
$$

Corollary 10. For $\|C\|<1$ and given positive real constants $h, h_{d}, r, r_{d}, \eta, \rho$, and $\beta$, system (54) is exponentially stable with a decay rate $k$, if there exist symmetric positive definite matrices $P_{j}$, any appropriate dimensional matrices $R_{s}, N_{i}, O_{i}$, 
$W_{i}, M_{j}$, and $G$, where $s=1,2, \ldots 6, i=1,2, \ldots, 9$, and $j=1,2, \ldots 10$, and positive real constants $\epsilon_{1}, \epsilon_{2}$ such that the following symmetric linear matrix inequalities hold:

$$
\begin{gathered}
{\left[\begin{array}{cc}
R_{1} & R_{2} \\
* & R_{3}
\end{array}\right]>0} \\
{\left[\begin{array}{cc}
R_{4} & R_{5} \\
* & R_{6}
\end{array}\right]>0} \\
{\left[\begin{array}{ccc}
e^{-2 k h} P_{3}-R_{3} & M_{1} & M_{2} \\
* & M_{3} & M_{4} \\
* & * & M_{5}
\end{array}\right] \geq 0,} \\
{\left[\begin{array}{ccc}
e^{-2 \beta k h} P_{6}-R_{6} & M_{6} & M_{7} \\
* & M_{8} & M_{9} \\
* & * & M_{10}
\end{array}\right] \geq 0} \\
\prod<0,
\end{gathered}
$$

where

$$
\Pi=\left[\begin{array}{ccccccccc}
\Sigma_{11} & \Sigma_{12} & \Sigma_{13} & \Sigma_{14} & \Sigma_{15} & \Sigma_{16} & \Sigma_{17} & \Sigma_{18} & \Sigma_{19} \\
* & \Sigma_{22} & \Sigma_{23} & \Sigma_{24} & \Sigma_{25} & \Sigma_{26} & \Sigma_{27} & \Sigma_{28} & \Sigma_{29} \\
* & * & \Sigma_{33} & \Sigma_{34} & \Sigma_{35} & \Sigma_{36} & \Sigma_{37} & \Sigma_{38} & \Sigma_{39} \\
* & * & * & \Sigma_{44} & \Sigma_{45} & \Sigma_{46} & \Sigma_{47} & \Sigma_{48} & \Sigma_{49} \\
* & * & * & * & \Sigma_{55} & \Sigma_{56} & \Sigma_{57} & \Sigma_{58} & \Sigma_{59} \\
* & * & * & * & * & \Sigma_{66} & \Sigma_{67} & \Sigma_{68} & \Sigma_{69} \\
* & * & * & * & * & * & \widetilde{\Sigma}_{77} & \Sigma_{78} & \Sigma_{79} \\
* & * & * & * & * & * & * & \Sigma_{88} & \Sigma_{89} \\
* & * & * & * & * & * & * & * & \Sigma_{99}
\end{array}\right]
$$

Moreover, the solution $x(t, \phi, \psi)$ satisfies the inequality

$$
\|x(t, \phi, \psi)\| \leq \sqrt{\frac{D}{\lambda_{\min }\left(P_{1}\right)}} \max [\|\phi\|,\|\psi\|] e^{-k t}, \quad \forall t \in R^{+},
$$

where $D=\lambda_{\text {max }}\left(P_{1}\right)+r \lambda_{\text {max }}\left(P_{2}\right)+h \lambda_{\text {max }}\left(P_{3}\right)+\beta h \lambda_{\text {max }}\left(P_{4}\right)+$ $h^{3} \lambda_{\max }\left(P_{5}+P_{7}+P_{9}\right)+(\beta h)^{3} \lambda_{\max }\left(P_{6}+P_{8}+P_{10}\right)+$ $h^{3} \lambda_{\max }\left(\left[\begin{array}{cc}R_{1} & R_{2} \\ * & R_{3}\end{array}\right]\right)+(\beta h)^{3} \lambda_{\max }\left(\left[\begin{array}{cc}R_{4} & R_{5} \\ * & R_{6}\end{array}\right]\right)$.

\section{Numerical Examples}

In order to show the effectiveness of the approaches presented in Section 3, three numerical examples are provided.

Example 1. Consider the robust exponential stability of system (1) with

$$
\begin{aligned}
& A(\alpha)=\alpha_{1}\left[\begin{array}{cc}
-2 & 0 \\
0 & -1
\end{array}\right]+\alpha_{2}\left[\begin{array}{cc}
-2 & 0 \\
0 & -0.8
\end{array}\right], \\
& B(\alpha)=\alpha_{1}\left[\begin{array}{cc}
-1 & 0 \\
-1 & -0.8
\end{array}\right]+\alpha_{2}\left[\begin{array}{cc}
-1 & 0 \\
-1 & -1
\end{array}\right],
\end{aligned}
$$

$$
\begin{gathered}
C(\alpha)=\alpha_{1}\left[\begin{array}{cc}
0.1 & 0 \\
0 & 0.1
\end{array}\right]+\alpha_{2}\left[\begin{array}{cc}
0.2 & 0 \\
0 & 0.1
\end{array}\right], \\
f(t, x(t))=\left[\begin{array}{c}
0.05 \sin (t) x_{1}(t) \\
0.05 \cos (t) x_{2}(t)
\end{array}\right], \\
g(t, x(t-h(t)))=\left[\begin{array}{c}
0.1 \cos (t)^{2} x_{1}(t-h(t)) \\
0.1 \sin (t)^{2} x_{2}(t-h(t))
\end{array}\right], \\
h(t)=0.9 \sin ^{2}\left(\frac{t}{2}\right), \\
w(t, \dot{x}(t-r(t)))=\left[\begin{array}{r}
0.1 \cos (t) \dot{x}_{1}(t-r(t)) \\
0.1 \sin (t) \dot{x}_{2}(t-r(t))
\end{array}\right], \\
r(t)=\cos ^{2}\left(\frac{t}{4}\right),
\end{gathered}
$$

where $x(t) \in R^{2}$. It is easy to see that $h=0.9, h_{d}=0.45$, $r=1, r_{d}=0.25, \eta=0.05, \rho=0.1, \gamma=0.1$, and given rate of convergence $k=0.1$. Decompose matrix $B(\alpha)$ as follows: $B(\alpha)=B_{1}(\alpha)+B_{2}(\alpha)$, where

$$
\begin{aligned}
& B_{1}(\alpha)=\alpha_{1}\left[\begin{array}{cc}
-0.83 & 0 \\
-1 & -0.63
\end{array}\right]+\alpha_{2}\left[\begin{array}{cc}
-0.83 & 0 \\
-1 & -0.83
\end{array}\right], \\
& B_{2}(\alpha)=\alpha_{1}\left[\begin{array}{cc}
-0.17 & 0 \\
0 & -0.17
\end{array}\right]+\alpha_{2}\left[\begin{array}{cc}
-0.17 & 0 \\
0 & -0.17
\end{array}\right]
\end{aligned}
$$

The numerical solutions $x_{1}(t)$ and $x_{2}(t)$ of system (1) with (58)-(59) are plotted in Figure 1 where the states $x_{1}(t)$ and $x_{2}(t)$ are attracted to the stable origin.

Solution. By using the LMI Toolbox in MATLAB (with accuracy 0.01), we use conditions (25) in Theorem 7 for system (1) with (58)-(59). The solutions of LMIs verify as follows:

$$
\begin{aligned}
P_{1}^{1} & =10^{3} \times\left[\begin{array}{cc}
3.3091 & -0.0455 \\
-0.0455 & 0.7941
\end{array}\right], \\
P_{2}^{1} & =10^{3} \times\left[\begin{array}{cc}
3.3091 & -0.0455 \\
-0.0455 & 0.7941
\end{array}\right], \\
P_{1}^{2} & =10^{3} \times\left[\begin{array}{cc}
4.3739 & -0.3929 \\
-0.3929 & 2.7610
\end{array}\right], \\
P_{2}^{2} & =10^{3} \times\left[\begin{array}{cc}
4.7896 & -0.4618 \\
-0.4618 & 2.8307
\end{array}\right], \\
P_{1}^{3} & =10^{4} \times\left[\begin{array}{cc}
1.5976 & 0.1384 \\
0.1384 & 0.3189
\end{array}\right], \\
P_{2}^{3} & =10^{4} \times\left[\begin{array}{cc}
1.5976 & 0.1384 \\
0.1384 & 0.3189
\end{array}\right], \\
P_{1}^{4} & =10^{3} \times\left[\begin{array}{cc}
9.8670 & -0.0635 \\
-0.0635 & 4.5726
\end{array}\right],
\end{aligned}
$$




$$
\begin{aligned}
& P_{2}^{4}=10^{3} \times\left[\begin{array}{cc}
8.8868 & -0.2421 \\
-0.2421 & 3.2473
\end{array}\right], \\
& P_{1}^{5}=10^{3} \times\left[\begin{array}{cc}
1.3009 & -0.1372 \\
-0.1372 & 0.6505
\end{array}\right] \text {, } \\
& P_{2}^{5}=10^{3} \times\left[\begin{array}{cc}
1.0898 & -0.0980 \\
-0.0980 & 0.3986
\end{array}\right] \text {, } \\
& P_{1}^{6}=10^{4} \times\left[\begin{array}{cc}
2.8885 & -0.0338 \\
-0.0338 & 2.6935
\end{array}\right] \text {, } \\
& P_{2}^{6}=10^{4} \times\left[\begin{array}{cc}
2.8526 & -0.0449 \\
-0.0449 & 2.4922
\end{array}\right] \text {, } \\
& P_{1}^{7}=10^{3} \times\left[\begin{array}{cc}
2.6064 & -0.3045 \\
-0.3045 & 2.5259
\end{array}\right] \text {, } \\
& P_{2}^{7}=10^{3} \times\left[\begin{array}{cc}
2.3663 & -0.1722 \\
-0.1722 & 2.5747
\end{array}\right] \text {, } \\
& P_{1}^{8}=10^{4} \times\left[\begin{array}{cc}
1.4801 & -0.0277 \\
-0.0277 & 1.4063
\end{array}\right] \text {, } \\
& P_{2}^{8}=10^{4} \times\left[\begin{array}{cc}
1.4740 & -0.0862 \\
-0.0862 & 1.4207
\end{array}\right] \text {, } \\
& P_{1}^{9}=10^{3} \times\left[\begin{array}{cc}
2.6064 & -0.3045 \\
-0.3045 & 2.5259
\end{array}\right] \text {, } \\
& P_{2}^{9}=10^{3} \times\left[\begin{array}{cc}
2.3663 & -0.1722 \\
-0.1722 & 2.5747
\end{array}\right] \text {, } \\
& P_{1}^{10}=10^{4} \times\left[\begin{array}{cc}
1.4570 & -0.0000 \\
-0.0000 & 1.2074
\end{array}\right] \text {, } \\
& P_{2}^{10}=10^{4} \times\left[\begin{array}{ll}
1.4354 & 0.0019 \\
0.0019 & 1.0978
\end{array}\right], \\
& M_{1}^{1}=10^{3} \times\left[\begin{array}{ll}
-9.1839 & -1.2537 \\
-1.2537 & -2.0706
\end{array}\right] \text {, } \\
& M_{2}^{1}=10^{4} \times\left[\begin{array}{ll}
-1.0642 & -0.1844 \\
-0.1844 & -0.2293
\end{array}\right] \text {, } \\
& M_{1}^{2}=10^{3} \times\left[\begin{array}{ll}
8.8459 & 1.1503 \\
1.1503 & 2.0066
\end{array}\right], \\
& M_{2}^{2}=10^{4} \times\left[\begin{array}{ll}
1.0288 & 0.1763 \\
0.1763 & 0.2238
\end{array}\right], \\
& M_{1}^{3}=10^{4} \times\left[\begin{array}{ll}
1.0499 & 0.1090 \\
0.1090 & 0.2928
\end{array}\right], \\
& M_{2}^{3}=10^{4} \times\left[\begin{array}{ll}
1.1838 & 0.1766 \\
0.1766 & 0.2881
\end{array}\right], \\
& M_{1}^{4}=10^{3} \times\left[\begin{array}{ll}
-8.8058 & -0.9604 \\
-0.9604 & -2.6545
\end{array}\right] \text {, } \\
& M_{2}^{4}=10^{4} \times\left[\begin{array}{ll}
-1.0454 & -0.1677 \\
-0.1677 & -0.2680
\end{array}\right] \text {, }
\end{aligned}
$$

$$
\begin{aligned}
& M_{1}^{5}=10^{4} \times\left[\begin{array}{ll}
1.0303 & 0.1018 \\
0.1018 & 0.3190
\end{array}\right] \text {, } \\
& M_{2}^{5}=10^{4} \times\left[\begin{array}{ll}
1.1590 & 0.1622 \\
0.1622 & 0.3078
\end{array}\right] \text {, } \\
& M_{1}^{6}=\left[\begin{array}{cc}
-34.9655 & -18.7513 \\
-18.7513 & 35.4129
\end{array}\right] \text {, } \\
& M_{2}^{6}=\left[\begin{array}{cc}
-39.8158 & -24.8476 \\
-24.8476 & 13.4547
\end{array}\right] \text {, } \\
& M_{1}^{7}=\left[\begin{array}{cc}
2.0904 & 2.7717 \\
2.7717 & -7.8109
\end{array}\right], \quad M_{2}^{7}=\left[\begin{array}{cc}
3.2803 & 5.2929 \\
5.2929 & -4.0693
\end{array}\right] \text {, } \\
& M_{1}^{8}=10^{4} \times\left[\begin{array}{ll}
1.4679 & 0.0003 \\
0.0003 & 1.2678
\end{array}\right], \\
& M_{2}^{8}=10^{4} \times\left[\begin{array}{cc}
1.4438 & -0.0080 \\
-0.0080 & 1.1830
\end{array}\right] \text {, } \\
& M_{1}^{9}=10^{3} \times\left[\begin{array}{cc}
-0.8905 & 0.0058 \\
0.0058 & -2.9028
\end{array}\right] \text {, } \\
& M_{2}^{9}=10^{3} \times\left[\begin{array}{ll}
-1.1307 & -0.0760 \\
-0.0760 & -3.7474
\end{array}\right] \text {, } \\
& M_{1}^{10}=10^{4} \times\left[\begin{array}{ll}
1.4684 & 0.0005 \\
0.0005 & 1.2673
\end{array}\right] \text {, } \\
& M_{2}^{10}=10^{4} \times\left[\begin{array}{cc}
1.4444 & -0.0077 \\
-0.0077 & 1.1828
\end{array}\right] \text {, } \\
& R_{1}^{1}=10^{3} \times\left[\begin{array}{ll}
2.9131 & 0.1748 \\
0.1748 & 0.7782
\end{array}\right] \text {, } \\
& R_{2}^{1}=10^{3} \times\left[\begin{array}{ll}
2.3463 & 0.0353 \\
0.0353 & 0.5806
\end{array}\right] \text {, } \\
& R_{1}^{2}=10^{3} \times\left[\begin{array}{ll}
1.1393 & 0.0531 \\
0.0531 & 0.1251
\end{array}\right], \\
& R_{2}^{2}=\left[\begin{array}{cc}
876.5627 & 32.5550 \\
32.5550 & 98.5599
\end{array}\right] \text {, } \\
& R_{1}^{3}=10^{3} \times\left[\begin{array}{cc}
2.6387 & -0.0759 \\
-0.0759 & 0.5866
\end{array}\right] \text {, } \\
& R_{2}^{3}=10^{3} \times\left[\begin{array}{cc}
2.0346 & -0.0762 \\
-0.0762 & 0.3589
\end{array}\right] \text {, } \\
& R_{1}^{4}=10^{3} \times\left[\begin{array}{cc}
5.6600 & -0.0415 \\
-0.0415 & 5.0541
\end{array}\right] \text {, } \\
& R_{2}^{4}=10^{3} \times\left[\begin{array}{cc}
5.5782 & -0.0710 \\
-0.0710 & 4.6031
\end{array}\right] \text {, } \\
& R_{1}^{5}=10^{4} \times\left[\begin{array}{cc}
-7.9071 & 0.0551 \\
0.0551 & -3.8199
\end{array}\right] \text {, } \\
& R_{2}^{5}=10^{4} \times\left[\begin{array}{cc}
-7.3912 & 0.4576 \\
0.4576 & -3.2145
\end{array}\right] \text {, }
\end{aligned}
$$




$$
\begin{aligned}
& R_{1}^{6}=10^{4} \times\left[\begin{array}{cc}
1.4893 & -0.0162 \\
-0.0162 & 1.3778
\end{array}\right], \\
& R_{2}^{6}=10^{4} \times\left[\begin{array}{cc}
1.4703 & -0.0223 \\
-0.0223 & 1.2713
\end{array}\right] \text {, } \\
& N_{1}^{1}=10^{3} \times\left[\begin{array}{cc}
8.7493 & 3.8534 \\
3.8534 & -3.1296
\end{array}\right] \text {, } \\
& N_{2}^{1}=10^{4} \times\left[\begin{array}{cc}
0.6691 & 0.2686 \\
0.2686 & -1.0299
\end{array}\right] \text {, } \\
& N_{1}^{2}=10^{3} \times\left[\begin{array}{cc}
-3.8698 & -0.3606 \\
-0.3606 & 1.4605
\end{array}\right] \text {, } \\
& N_{2}^{2}=10^{3} \times\left[\begin{array}{cc}
-4.5981 & -0.9753 \\
-0.9753 & 1.2772
\end{array}\right] \text {, } \\
& N_{1}^{3}=\left[\begin{array}{ll}
-114.8946 & -186.6948 \\
-186.6948 & -113.8034
\end{array}\right] \text {, } \\
& N_{2}^{3}=\left[\begin{array}{cc}
210.0606 & -115.9077 \\
-115.9077 & -174.4056
\end{array}\right] \text {, } \\
& N_{1}^{4}=10^{4} \times\left[\begin{array}{cc}
-1.1296 & -0.0266 \\
-0.0266 & 0.1446
\end{array}\right] \text {, } \\
& N_{2}^{4}=10^{4} \times\left[\begin{array}{cc}
-1.0062 & 0.1041 \\
0.1041 & 0.8782
\end{array}\right] \text {, } \\
& N_{1}^{5}=10^{3} \times\left[\begin{array}{cc}
4.5589 & -2.3847 \\
-2.3847 & 0.8561
\end{array}\right] \text {, } \\
& N_{2}^{5}=10^{3} \times\left[\begin{array}{cc}
5.3887 & -2.4363 \\
-2.4363 & 1.1619
\end{array}\right] \text {, } \\
& N_{1}^{6}=10^{3} \times\left[\begin{array}{cc}
5.9564 & -1.9530 \\
-1.9530 & 1.5688
\end{array}\right] \text {, } \\
& N_{2}^{6}=10^{3} \times\left[\begin{array}{cc}
6.7696 & -1.9958 \\
-1.9958 & 2.0384
\end{array}\right] \text {, } \\
& N_{1}^{7}=10^{3} \times\left[\begin{array}{cc}
5.9602 & -0.2891 \\
-0.2891 & 2.9332
\end{array}\right] \text {, } \\
& N_{2}^{7}=10^{3} \times\left[\begin{array}{cc}
6.3929 & -0.0855 \\
-0.0855 & 3.1668
\end{array}\right] \text {, } \\
& N_{1}^{8}=10^{3} \times\left[\begin{array}{cc}
-9.6508 & -0.4780 \\
-0.4780 & 3.5901
\end{array}\right] \text {, } \\
& N_{2}^{8}=10^{4} \times\left[\begin{array}{cc}
-0.8505 & 0.0574 \\
0.0574 & 1.1344
\end{array}\right] \text {, } \\
& N_{1}^{9}=10^{3} \times\left[\begin{array}{cc}
5.5789 & -2.0704 \\
-2.0704 & 1.3737
\end{array}\right] \text {, } \\
& N_{2}^{9}=10^{3} \times\left[\begin{array}{cc}
6.3923 & -2.1169 \\
-2.1169 & 1.8008
\end{array}\right] \text {, } \\
& N_{1}^{10}=10^{3} \times\left[\begin{array}{cc}
-3.6595 & 3.2032 \\
3.2032 & -0.1648
\end{array}\right] \text {, }
\end{aligned}
$$

$$
\begin{aligned}
& N_{2}^{10}=10^{3} \times\left[\begin{array}{cc}
-4.3781 & 3.2120 \\
3.2120 & 0.1271
\end{array}\right], \\
& O_{1}^{1}=10^{4} \times\left[\begin{array}{cc}
1.5923 & -0.7669 \\
-0.7669 & 1.6350
\end{array}\right] \text {, } \\
& O_{2}^{1}=10^{4} \times\left[\begin{array}{cc}
1.8134 & -0.6324 \\
-0.6324 & 2.3112
\end{array}\right] \text {, } \\
& O_{1}^{2}=10^{4} \times\left[\begin{array}{cc}
1.0371 & -0.0001 \\
-0.0001 & -0.3635
\end{array}\right] \text {, } \\
& O_{2}^{2}=10^{4} \times\left[\begin{array}{cc}
0.9152 & -0.1464 \\
-0.1464 & -1.0978
\end{array}\right] \text {, } \\
& O_{1}^{3}=10^{3} \times\left[\begin{array}{cc}
-1.0355 & 0.1515 \\
0.1515 & -0.3626
\end{array}\right] \text {, } \\
& O_{2}^{3}=10^{3} \times\left[\begin{array}{cc}
-1.4990 & 0.3148 \\
0.3148 & -0.4988
\end{array}\right] \text {, } \\
& O_{1}^{4}=\left[\begin{array}{cc}
-51.1797 & 71.7167 \\
71.7167 & -390.4844
\end{array}\right] \text {, } \\
& O_{2}^{4}=\left[\begin{array}{cc}
-22.7818 & -177.9764 \\
-177.9764 & 237.5644
\end{array}\right] \text {, } \\
& O_{1}^{5}=10^{4} \times\left[\begin{array}{cc}
-1.4569 & 0.2376 \\
0.2376 & -0.4763
\end{array}\right] \text {, } \\
& O_{2}^{5}=10^{4} \times\left[\begin{array}{cc}
-1.5078 & 0.2277 \\
0.2277 & -0.4312
\end{array}\right] \text {, } \\
& O_{1}^{6}=10^{4} \times\left[\begin{array}{cc}
-1.3667 & 0.2475 \\
0.2475 & -0.4459
\end{array}\right] \text {, } \\
& O_{2}^{6}=10^{4} \times\left[\begin{array}{cc}
-1.4169 & 0.2441 \\
0.2441 & -0.4015
\end{array}\right] \text {, } \\
& O_{1}^{7}=10^{3} \times\left[\begin{array}{cc}
5.5038 & 0.7900 \\
0.7900 & -4.7294
\end{array}\right] \text {, } \\
& \mathrm{O}_{2}^{7}=10^{4} \times\left[\begin{array}{cc}
0.4206 & -0.0170 \\
-0.0170 & -1.2249
\end{array}\right] \text {, } \\
& O_{1}^{8}=\left[\begin{array}{cc}
244.5684 & 88.4596 \\
88.4596 & 481.2263
\end{array}\right] \text {, } \\
& O_{2}^{8}=\left[\begin{array}{cc}
272.5078 & 401.6259 \\
401.6259 & -0.2695
\end{array}\right] \text {, } \\
& O_{1}^{9}=10^{4} \times\left[\begin{array}{cc}
-1.3911 & 0.2447 \\
0.2447 & -0.4543
\end{array}\right] \text {, } \\
& O_{2}^{9}=10^{4} \times\left[\begin{array}{cc}
-1.4415 & 0.2394 \\
0.2394 & -0.4095
\end{array}\right] \text {, } \\
& O_{1}^{10}=10^{4} \times\left[\begin{array}{cc}
1.3674 & -0.2535 \\
-0.2535 & 0.3868
\end{array}\right], \\
& O_{2}^{10}=10^{4} \times\left[\begin{array}{cc}
1.4277 & -0.2479 \\
-0.2479 & 0.3580
\end{array}\right] \text {, }
\end{aligned}
$$




$$
\begin{aligned}
& W_{1}^{1}=10^{4} \times\left[\begin{array}{cc}
1.5106 & -0.0468 \\
-0.0468 & 0.7929
\end{array}\right] \text {, } \\
& W_{2}^{1}=10^{4} \times\left[\begin{array}{cc}
1.4523 & -0.0341 \\
-0.0341 & 0.7069
\end{array}\right] \text {, } \\
& W_{1}^{2}=10^{3} \times\left[\begin{array}{cc}
5.9653 & -2.4134 \\
-2.4134 & 1.7851
\end{array}\right] \text {, } \\
& W_{2}^{2}=10^{3} \times\left[\begin{array}{cc}
6.9091 & -2.5666 \\
-2.5666 & 1.9597
\end{array}\right] \text {, } \\
& W_{1}^{3}=\left[\begin{array}{cc}
-289.7085 & 24.4200 \\
24.4200 & -461.3886
\end{array}\right] \text {, } \\
& W_{2}^{3}=\left[\begin{array}{cc}
-223.5193 & 93.6566 \\
93.6566 & -234.3346
\end{array}\right] \text {, } \\
& W_{1}^{4}=10^{4} \times\left[\begin{array}{cc}
-1.2746 & 0.2653 \\
0.2653 & -0.3694
\end{array}\right] \text {, } \\
& W_{2}^{4}=10^{4} \times\left[\begin{array}{cc}
-1.3242 & 0.2636 \\
0.2636 & -0.3371
\end{array}\right] \text {, } \\
& W_{1}^{5}=10^{3} \times\left[\begin{array}{cc}
4.8778 & -0.0916 \\
-0.0916 & 3.8521
\end{array}\right] \text {, } \\
& W_{2}^{5}=10^{3} \times\left[\begin{array}{cc}
4.8245 & -0.0681 \\
-0.0681 & 3.9058
\end{array}\right] \text {, } \\
& W_{1}^{6}=10^{3} \times\left[\begin{array}{cc}
7.9418 & -0.4415 \\
-0.4415 & 6.9527
\end{array}\right] \text {, } \\
& W_{2}^{6}=10^{3} \times\left[\begin{array}{cc}
7.9864 & -0.4713 \\
-0.4713 & 7.2384
\end{array}\right] \text {, } \\
& W_{1}^{7}=10^{3} \times\left[\begin{array}{cc}
4.5999 & -2.3966 \\
-2.3966 & 0.1608
\end{array}\right] \text {, } \\
& W_{2}^{7}=10^{3} \times\left[\begin{array}{cc}
5.2537 & -2.3858 \\
-2.3858 & 0.0285
\end{array}\right] \text {, } \\
& W_{1}^{8}=10^{4} \times\left[\begin{array}{cc}
-1.3844 & 0.2577 \\
0.2577 & -0.4094
\end{array}\right] \text {, } \\
& W_{2}^{8}=10^{4} \times\left[\begin{array}{cc}
-1.4426 & 0.2499 \\
0.2499 & -0.3763
\end{array}\right] \text {, } \\
& W_{1}^{9}=10^{3} \times\left[\begin{array}{cc}
7.1086 & -0.3474 \\
-0.3474 & 6.1090
\end{array}\right] \text {, } \\
& W_{2}^{9}=10^{3} \times\left[\begin{array}{cc}
7.1257 & -0.3627 \\
-0.3627 & 6.3346
\end{array}\right] \text {, } \\
& W_{1}^{10}=10^{3} \times\left[\begin{array}{cc}
8.0468 & -0.7400 \\
-0.7400 & 6.5133
\end{array}\right] \text {, } \\
& W_{2}^{10}=10^{3} \times\left[\begin{array}{cc}
8.1365 & -0.8020 \\
-0.8020 & 6.8109
\end{array}\right] \text {, } \\
& G=\left[\begin{array}{cc}
-2.1527 & 1.4389 \\
6.5441 & -11.7139
\end{array}\right], \quad \epsilon_{1}=4.2164 \times 10^{4}, \\
& \epsilon_{2}=5.7269 \times 10^{4}, \quad \epsilon_{3}=5.3163 \times 10^{4} .
\end{aligned}
$$

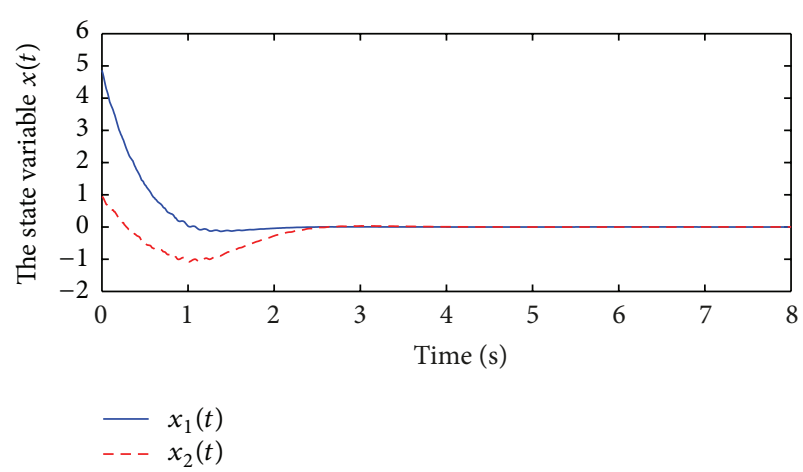

FIGURE 1: The simulation solutions $x_{1}(t)$ and $x_{2}(t)$ are presented for system (1) with (58)-(59) in Example 1, $\alpha_{1}=\alpha_{2}=1 / 2$, and initial conditions $x_{1}(t)=2+3 \cos (t), x_{2}(t)=1+2 \sin (t), t \in[-1,0]$, by using the Runge-Kutta fourth order method with Matlab.

TABLE 1: The maximum allowed time delay $h$ for $k=0.1, \eta=0.05$, $\rho=0.1$, and $\beta=0.1$.

\begin{tabular}{lccc}
\hline$h_{d}=r_{d}$ & 0 & 0.5 & 0.9 \\
\hline Chen et al. (2008) [3] & 1.2999 & 0.9442 & 0.5471 \\
Qiu and Cui (2010) [26] & 1.4008 & 1.0120 & 0.6438 \\
Pinjai and Mukdasai (2011) [25] & 1.6237 & 1.1052 & 0.6205 \\
Corollary 10 & 6.4417 & 5.2362 & 2.5666 \\
\hline
\end{tabular}

Example 2. Consider the following neutral system (54), which is considered in $[3,25,26]$ :

$$
\begin{aligned}
\dot{x}(t)= & A x(t)+B x(t-h(t))+C \dot{x}(t-r(t)) \\
& +f(t, x(t))+g(t, x(t-h(t))),
\end{aligned}
$$

with

$$
A=\left[\begin{array}{cc}
-2 & 0 \\
0 & -0.9
\end{array}\right], \quad B=\left[\begin{array}{cc}
-1 & 0 \\
-1 & -1
\end{array}\right], \quad C=\left[\begin{array}{cc}
0.1 & 0 \\
0 & 0.1
\end{array}\right] .
$$

Decompose matrix $B$ as follows: $B=B_{1}+B_{2}$, where

$$
B_{1}=\left[\begin{array}{cc}
-0.83 & 0 \\
-1 & -0.83
\end{array}\right], \quad B_{2}=\left[\begin{array}{cc}
-0.17 & 0 \\
0 & -0.17
\end{array}\right],
$$

$\|f(t, x(t))\| \leq \eta\|x(t)\|$, and $\|g(t, x(t-h(t)))\| \leq \rho\|x(t-h(t))\|$. The maximum value $h$ for exponential stability of system (61) with (62)-(63) is listed in the comparison in Table 1, for different values of $h_{d}$ and $r_{d}$. In Table 1 , we let $\eta=0.05$, $\rho=0.1, \beta=0.1$ and $h(t)=r(t)$. We can see that our results in Corollary 8 are much less conservative than in $[3,25,26]$.

Example 3. Consider the following neutral system (51), which is considered in [14, 28]:

$$
\begin{aligned}
\dot{x}(t)= & A x(t)+B x(t-h(t))+C \dot{x}(t-r(t))+f(t, x(t)) \\
& +g(t, x(t-h(t)))+w(t, \dot{x}(t-r(t))),
\end{aligned}
$$


TABLE 2: The maximum allowed time delay $h$.

\begin{tabular}{|c|c|c|c|c|c|}
\hline$h_{d}=r_{d}=0$ & $k=0.1$ & $k=0.3$ & $k=0.5$ & $k=0.7$ & $k=0.9$ \\
\hline Syed Ali (2012) [28] & 10.2180 & 2.9481 & 1.4126 & 0.7232 & 0.3045 \\
\hline Liu et al. (2013) [14] & 12.2475 & 3.7460 & 1.9563 & 1.1015 & 0.5957 \\
\hline Corollary 9 & 14.1728 & 4.7242 & 2.8345 & 2.0246 & 1.5747 \\
\hline$h_{d}=r_{d}=0.5$ & $k=0.1$ & $k=0.3$ & $k=0.5$ & $k=0.7$ & $k=0.9$ \\
\hline Syed Ali (2012) [28] & 6.7523 & 1.7922 & 0.7308 & 0.3580 & 0.1027 \\
\hline Liu et al. (2013) [14] & 10.8211 & 3.3202 & 1.7390 & 0.9662 & 0.4857 \\
\hline Corollary 9 & 11.5872 & 3.8624 & 2.3174 & 1.6553 & 1.2874 \\
\hline
\end{tabular}

with

$$
A=\left[\begin{array}{cc}
-2 & 0 \\
0 & -2
\end{array}\right], \quad B=\left[\begin{array}{cc}
0 & 0.4 \\
0.4 & 0
\end{array}\right], \quad C=\left[\begin{array}{cc}
0.1 & 0 \\
0 & 0.1
\end{array}\right] .
$$

Decompose matrix $B$ as follows: $B=B_{1}+B_{2}$, where

$$
B_{1}=\left[\begin{array}{cc}
0 & 0.3 \\
0.2 & 0
\end{array}\right], \quad B_{2}=\left[\begin{array}{cc}
0 & 0.1 \\
0.2 & 0
\end{array}\right],
$$

$\|f(t, x(t))\| \leq \eta\|x(t)\|,\|g(t, x(t-h(t)))\| \leq \rho\|x(t-h(t))\|$, and $\|w(t, \dot{x}(t-r(t)))\| \leq \gamma\|\dot{x}(t-r(t))\|$. By Corollary 9 to system (64) with (65)-(66), one can obtain the maximum upper bounds of the time delay with different convergence rate $k$ as listed in Table 2. In Table 2, we let $h=0.1, \eta=0.1$, $\rho=0.05, \gamma=0.05$, and $\beta=0.1$. It is clear that the results in Corollary 9 give larger delay bounds than the recent results in $[14,28]$.

\section{Conclusions}

The problem of robust exponential stability for LPD neutral systems with mixed time-varying delays and nonlinear uncertainties has been presented. Based on combination of Leibniz-Newton formula, free-weighting matrices, linear matrix inequality, Cauchy's inequality, modified version of Jensen's inequality, model transformation, and the use of suitable parameter-dependent Lyapunov-Krasovskii functional, new delay-dependent robust exponential stability criteria are formulated in terms of LMIs. Numerical examples have shown significant improvements over some existing results.

\section{Acknowledgments}

This work was supported by the Higher Education Research Promotion and the National Research University Project of Thailand, Office of the Higher Education Commission, through the Cluster of Research to Enhance the Quality of Basic Education, Khon Kaen University, Khon Kaen, Thailand.

\section{References}

[1] K. Gu, V. L. Kharitonov, and J. Chen, Stability of Time-Delay System, Birkhäauser, Berlin, Germany, 2003.

[2] Y. Y. Cao and J. Lam, "Computation of robust stability bounds for time-delay systems with nonlinear time-varying perturbations," International Journal of Systems Science, vol. 31, pp. 359-365, 2000.

[3] Y. Chen, A. Xue, R. Lu, and S. Zhou, "On robustly exponential stability of uncertain neutral systems with time-varying delays and nonlinear perturbations," Nonlinear Analysis: Theory, Methods \& Applications, vol. 68, no. 8, pp. 2464-2470, 2008.

[4] W.-H. Chen and W. X. Zheng, "Delay-dependent robust stabilization for uncertain neutral systems with distributed delays," Automatica, vol. 43, no. 1, pp. 95-104, 2007.

[5] J. Hale, Theory of Functional Differential Equations, Springer, New York, NY, USA, 2nd edition, 1977.

[6] Y. He, M. Wu, J.-H. She, and G.-P. Liu, "Parameter-dependent Lyapunov functional for stability of time-delay systems with polytopic-type uncertainties," IEEE Transactions on Automatic Control, vol. 49, no. 5, pp. 828-832, 2004.

[7] Y. He, Q.-G. Wang, C. Lin, and M. Wu, "Delay-range-dependent stability for systems with time-varying delay," Automatica, vol. 43, no. 2, pp. 371-376, 2007.

[8] X. Jiang and Q.-L. Han, "On $H_{\infty}$ control for linear systems with interval time-varying delay," Automatica, vol. 41, no. 12, pp. 2099-2106, 2005.

[9] V. L. Kharitonov and D. Hinrichsen, "Exponential estimates for time delay systems," Systems \& Control Letters, vol. 53, no. 5, pp. 395-405, 2004.

[10] O. M. Kwon and J. H. Park, "Exponential stability for timedelay systems with interval time-varying delays and nonlinear perturbations," Journal of Optimization Theory and Applications, vol. 139, no. 2, pp. 277-293, 2008.

[11] O. M. Kwon, J. H. Park, and S. M. Lee, "On robust stability criterion for dynamic systems with time-varying delays and nonlinear perturbations," Applied Mathematics and Computation, vol. 203, no. 2, pp. 937-942, 2008.

[12] C. Li, J. Sun, and R. Sun, "Stability analysis of a class of stochastic differential delay equations with nonlinear impulsive effects," Journal of the Franklin Institute, vol. 347, no. 7, pp. 1186-1198, 2010.

[13] C. Li, J. Shi, and J. Sun, "Stability of impulsive stochastic differential delay systems and its application to impulsive stochastic neural networks," Nonlinear Analysis: Theory, Methods \& Applications, vol. 74, no. 10, pp. 3099-3111, 2011.

[14] Y. Liu, S. M. Lee, O. M. Kwon, and J. H. Park, "Delay-dependent exponential stability criteria for 9 neutral systems with interval time-varying delay and nonlinear perturbations," Journal of Franklin Institute, vol. 350, no. 10, pp. 3313-3327, 2013.

[15] Y. S. Moon, P. Park, W. H. Kwon, and Y. S. Lee, "Delaydependent robust stabilization of uncertain state-delayed systems," International Journal of Control, vol. 74, no. 14, pp. 14471455, 2001. 
[16] S. Mondié and V. L. Kharitonov, "Exponential estimates for retarded time-delay systems: an LMI approach," IEEE Transactions on Automatic Control, vol. 50, no. 2, pp. 268-273, 2005.

[17] K. Mukdasai and P. Niamsup, "Robust stability of discrete-time linear parameter dependent system with delay," Thai Journal of Mathematics, vol. 8, no. 4, pp. 11-20, 2010.

[18] K. Mukdasai, "Robust exponential stability for LPD discretetime system with interval time-varying delay," Journal of Applied Mathematics, vol. 2013, Article ID 237430, 13 pages, 2012.

[19] K. Mukdasai, A. Wongphat, and P. Niamsup, "Robust exponential stability criteria of LPD systems with mixed time-varying delays and nonlinear perturbations," Abstract and Applied Analysis, vol. 2012, Article ID 348418, 20 pages, 2012.

[20] P. T. Nam, "Exponential stability criterion for time-delay systems with nonlinear uncertainties," Applied Mathematics and Computation, vol. 214, no. 2, pp. 374-380, 2009.

[21] P. Niamsup, K. Mukdasai, and V. N. Phat, "Improved exponential stability for time-varying systems with nonlinear delayed perturbations," Applied Mathematics and Computation, vol. 204, no. 1, pp. 490-495, 2008.

[22] P. Niamsup and V. N. Phat, " $H_{\infty}$ control for nonlinear timevarying delay systems with convex polytopic uncertainties," Nonlinear Analysis: Theory, Methods \& Applications, vol. 72, no. 11, pp. 4254-4263, 2010.

[23] J. H. Park and O. Kwon, "Novel stability criterion of time delay systems with nonlinear uncertainties," Applied Mathematics Letters, vol. 18, no. 6, pp. 683-688, 2005.

[24] V. N. Phat and P. Niamsup, "Stability of linear time-varying delay systems and applications to control problems," Journal of Computational and Applied Mathematics, vol. 194, no. 2, pp. 343-356, 2006.

[25] S. Pinjai and K. Mukdasai, "New robust exponential stability criterion for uncertain neutral systems with discrete and distributed time-varying delays and nonlinear perturbations," Abstract and Applied Analysis, vol. 2011, Article ID 463603, 16 pages, 2011.

[26] F. Qiu and B. T. Cui, "Improved exponential stability criteria for uncertain neutral system with nonlinear parameter perturbations," International Journal of Automation and Computing, vol. 7, no. 4, pp. 413-418, 2010.

[27] F. Qiu, B. Cui, and Y. Ji, "Further results on robust stability of neutral system with mixed time-varying delays and nonlinear perturbations," Nonlinear Analysis: Real World Applications, vol. 11, no. 2, pp. 895-906, 2010.

[28] M. Syed Ali, "On exponential stability of neutral delay differential system with nonlinear uncertainties," Communications in Nonlinear Science and Numerical Simulation, vol. 17, no. 6, pp. 2595-2601, 2012.

[29] L. Xiong, S. Zhong, and D. Li, "Novel delay-dependent asymptotical stability of neutral systems with nonlinear perturbations," Journal of Computational and Applied Mathematics, vol. 232, no. 2, pp. 505-513, 2009.

[30] Y. Xia and Y. Jia, "Robust stability functionals of state delayed systems with polytopic type uncertainties via parameter dependent Lyapunov functions," International Journal of Control, vol. 75, no. 16-17, pp. 1427-1434, 2002.

[31] W.-A. Zhang and L. Yu, "Delay-dependent robust stability of neutral systems with mixed delays and nonlinear perturbations," Acta Automatica Sinica, vol. 33, no. 8, pp. 863-866, 2007.
[32] W. Zhang, X.-S. Cai, and Z.-Z. Han, "Robust stability criteria for systems with interval time-varying delay and nonlinear perturbations," Journal of Computational and Applied Mathematics, vol. 234, no. 1, pp. 174-180, 2010.

[33] X.-M. Zhang, M. Wu, J.-H. She, and Y. He, "Delay-dependent stabilization of linear systems with time-varying state and input delays," Automatica, vol. 41, no. 8, pp. 1405-1412, 2005.

[34] X.-L. Zhu and G.-H. Yang, "Delay-dependent stability criteria for systems with differentiable time delays," Acta Automatica Sinica, vol. 34, no. 7, pp. 765-771, 2008.

[35] Z. Zuo and Y. Wang, "New stability criterion for a class of linear systems with time-varying delay and nonlinear perturbations," IEE Proceedings, vol. 153, no. 5, pp. 623-626, 2006. 


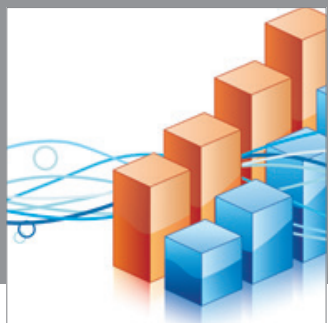

Advances in

Operations Research

mansans

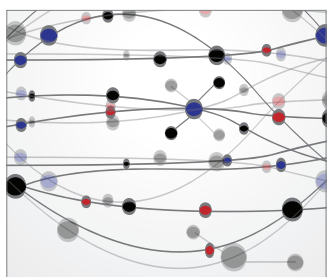

The Scientific World Journal
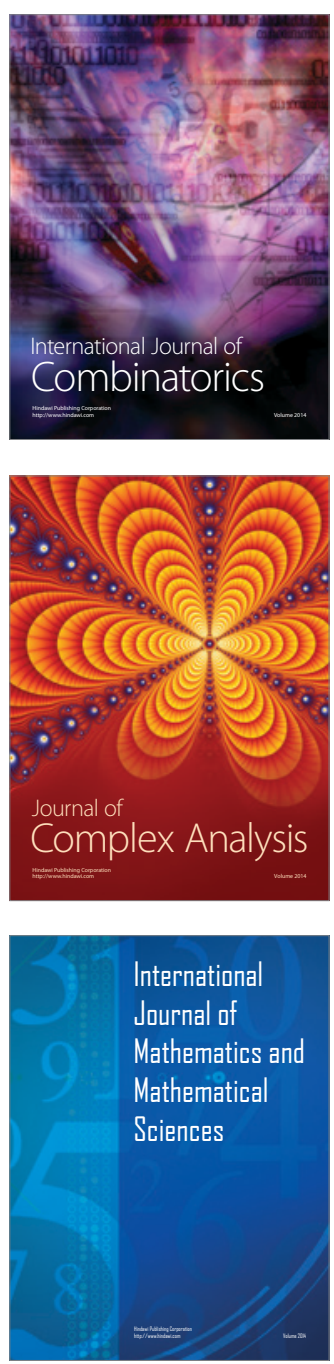
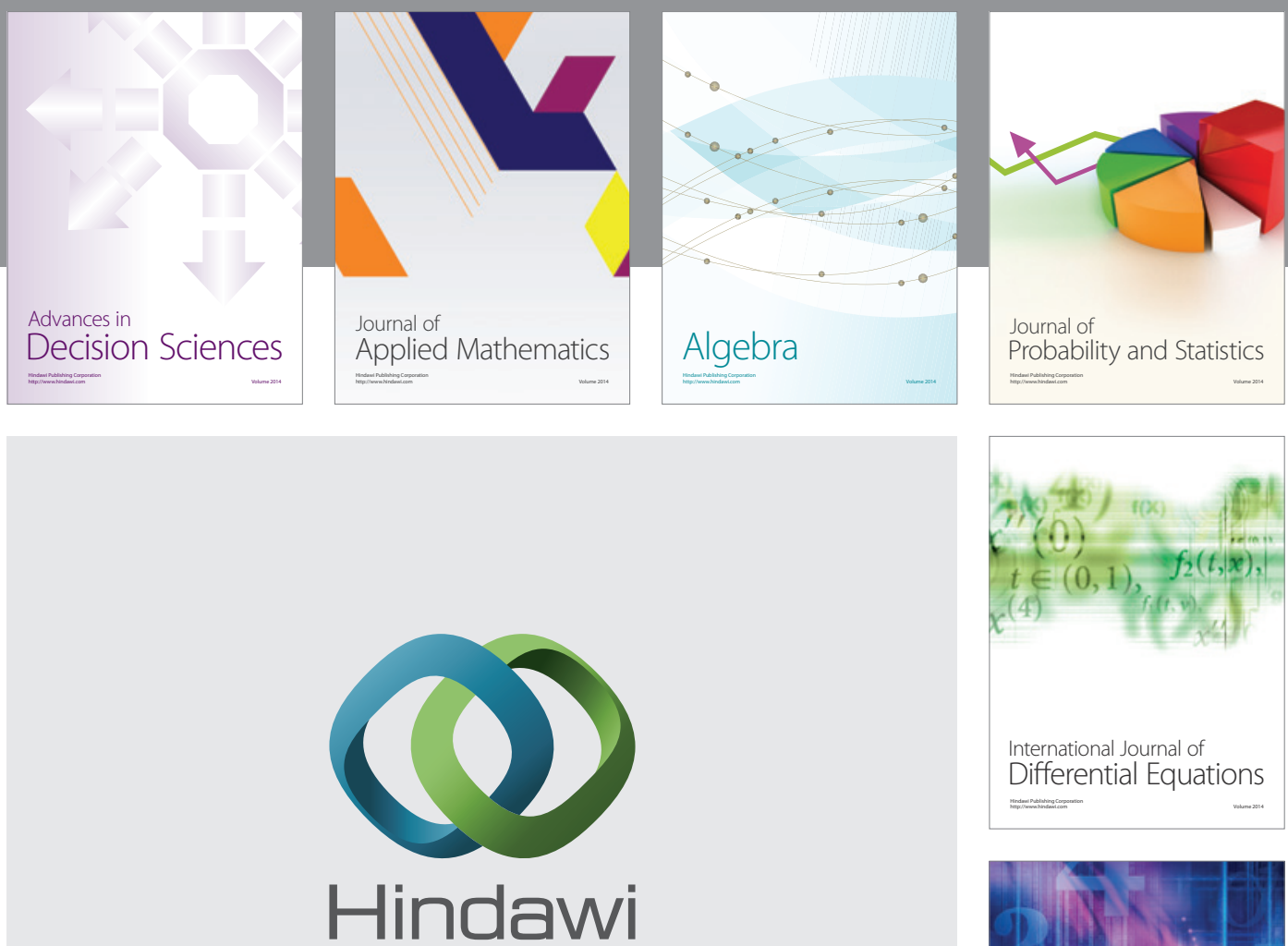

Submit your manuscripts at http://www.hindawi.com
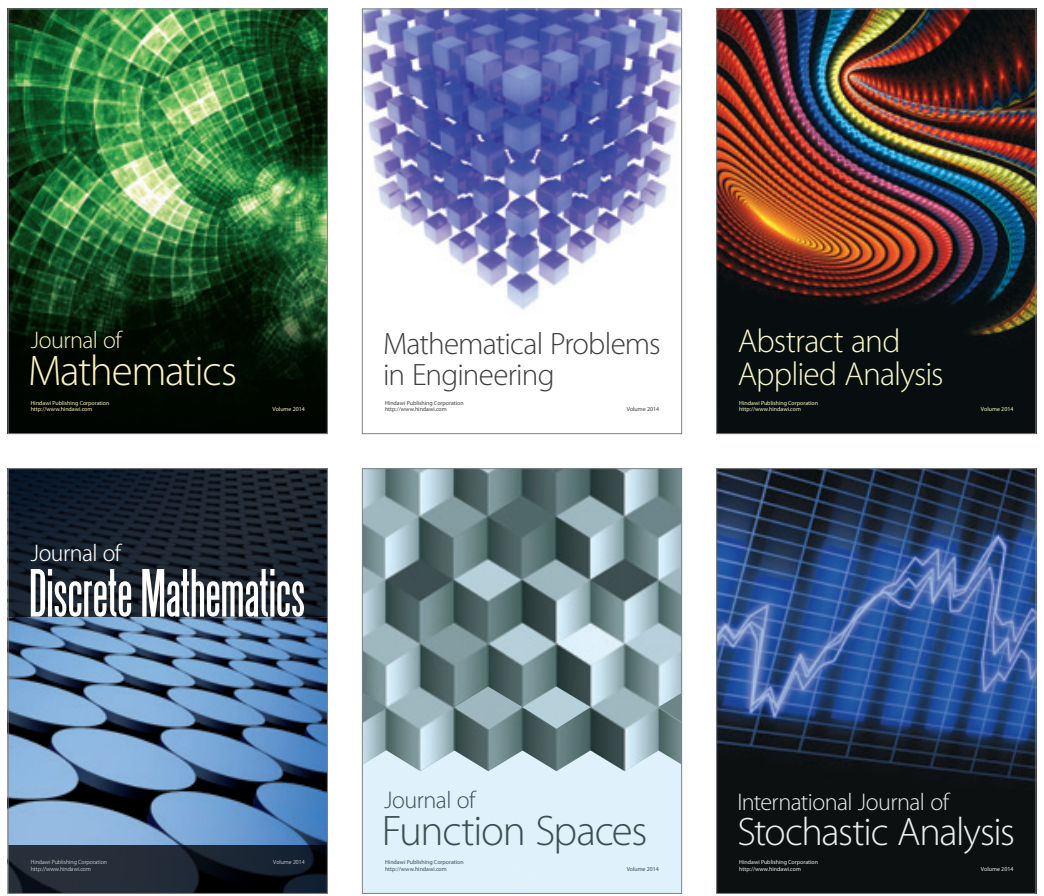

Journal of

Function Spaces

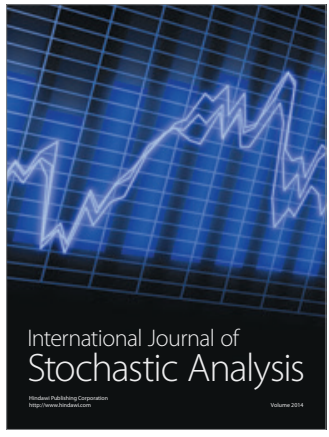

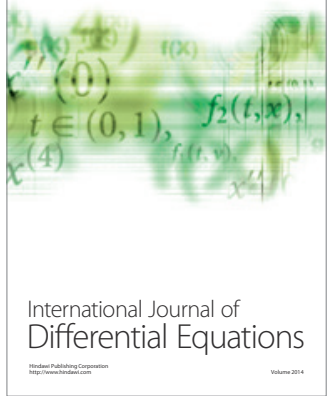
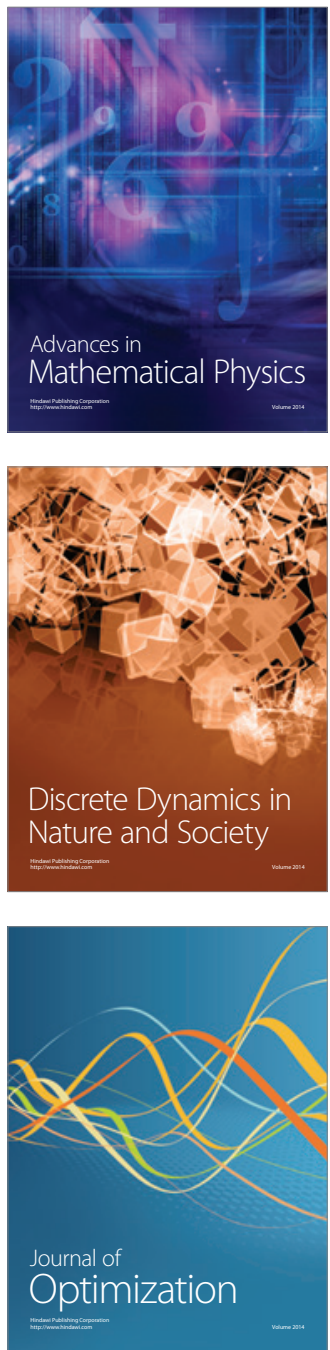